\title{
Reliability and dimensionality of judgments of visually textured materials
}

\author{
RAYMOND Y. CHO, VICKY YANG, and PETER E. HALLETT \\ University of Toronto, Toronto, Ontario, Canada
}

\begin{abstract}
We extended perceptual studies of the Brodatz set of textured materials. In the experiments, texture perception for different texture sets, viewing distances, or lighting intensities was examined. Subjects compared one pair of textures at a time. The main task was to rapidly rate all of the texture pairs on a number scale for their overall dissimilarities first and then for their dissimilarities according to six specified attributes (e.g., texture contrast). The implied dimensionality of perceptual texture space was usually at least four, rather than three. All six attributes proved to be useful predictors of overall dissimilarity, especially coarseness and regularity. The novel attribute texture lightness, an assessment of mean surface reflectance, was important when viewing conditions were wide-ranging. We were impressed by the general validity of texture judgments across subject, texture set, and comfortable viewing distances or lighting intensities. The attributes are nonorthogonal directions in four-dimensional perceptual space and are probably not narrow linear axes. In a supplementary experiment, we studied a completely different task: identifying textures from a distance. The dimensionality for this more refined task is similar to that for rating judgments, so our findings may have general application.
\end{abstract}

It has been suggested that the perception of texture is inherently a process of comparison, whether between two or more simultaneously presented stimuli or between stimulus and memory representations (Harvey \& Gervais, 1981). What can be said about the perceptual space that represents such judgments?

Most studies to date have employed simple artificial textures and gratings as stimuli. Richards and Polit (1974) assumed the existence of independent spatial filters (Campbell \& Robson, 1968) and used a four-filter model to explain texture matches of noisy one-dimensional (1-D) sine-wave gratings. Employing similar textures, Harvey and Gervais $(1978,1981)$ predicted comparative judgments from either the textures' spatial frequency components or the responses of four spatial filters. Kahana and Bennett (1994) used a two-channel model of phase discrimination to explain comparisons of perceived similarity between $f+2 f$ gratings (fundamental and second harmonic). Julesz (1981) tried to explain the preattentive perception of patterns of simple repeated elements by the

This work was supported by grants from the Medical Research Council of Canada to P.E.H. and from the National Sciences and Engineering Research Council to R.Y.C. Thanks to A. Bendzsack and H. Nikmanesh for their careful replications of the varying luminance and distance experiments. R. J. Tibshirani (Department of Statistics and Biostatistics, University of Toronto) performed the nonlinear multiple regressions. Thanks to R. J. Tibshirani, B. Schneider, and P. Bennett (Department of Psychology, University of Toronto) for reading the manuscript. V.Y. is in the Department of Electrical and Computer Engineering, and P.E.H. is in the Departments of Physiology and of Zoology, Biomedical Engineering, and Ophthalmology, at Toronto. Correspondence concerning this article should be addressed to $\mathrm{R}$. Y. Cho (now at the University of Pittsburgh and Princeton University), Western Psychiatric Institute and Clinic, University of Pittsburgh, Pittsburgh, PA 15213 (e-mail: chory@msx.upmc.edu). statistics of local spatial features, or textons, such as corners and intersections. But such approaches have been criticized for lacking general applicability. Malik and Perona (1990) provided one instance of a more comprehensive spatial filter model motivated by physiological findings, which included outputs of simple cells, local inhibitory mechanisms, and boundary detection. Hofmann and Hallett (1993a, 1993b) explained rating comparisons of three component phase textures with a particularly economical model of the early visual cortex.

It may not be unexpected that the outputs of simple spatial frequency and phase discrimination filters are successful at explaining the perceived similarities of simple synthetic textures that differ only in the characteristics of a relatively small number of component gratings. The interest of this study, however, is in the characterization of rapid judgments of the much more complex, natural textures that are encountered in ecological conditions. What attributes are used in judgments of natural textures has not been firmly established. And is a texture judgment essentially an objective process - that is, one insensitive to observer idiosyncrasy, choice of texture, task, or viewing condition? Color science faced similar challenges and managed to capture the regularities of color processing in a three-dimensional (3-D) color space (see, e.g., Boynton \& Olson, 1987; Cronly-Dillon \& Gouras, 1991; Uchikawa \& Boynton, 1987). Although there have been several claims of similarity between texture perception and color vision (Rao \& Lohse, 1996; Richards, 1979), it is far from clear that this is more than a hoped for analogy. As of yet, there is no standard texture space, and major issues concerning the dimensionality and axes of such a space and concerning its universality or its sensitivity to personal idiosyncrasies have yet to be resolved. 
Texture perception could, in principle, involve a very high dimensionality, in the light of some neurophysiological and psychophysical evidence. At each point of the striate cortex, there exist many different flavors of simple cells (e.g., about 5 spatial frequency bands $\times 6$ orientations $\times$ 4 phases $=$ on the order of 100-200 cell types; see, e.g., Daugman, 1987; De Valois, Albrecht, \& Thorell, 1982; Field, 1987; Fleet \& Jepson, 1989; Sakitt \& Barlow, 1982). A perceptual study by Richards (1979) posited the existence of six spatial frequency channels and six orientation channels and proposed that perceptually similar textures elicited similar responses in all of these channels. It might seem that many, or even very many, channels are necessary to accurately characterize a small patch of texture.

It is quite possible, however, that not all the information carried by the high-dimensional sensory representation is preserved in rapid innate judgments of natural textures. A number of studies have used higher order features to explain relatively coarse judgments of the similarities in the appearances of the textures found in the Brodatz (1966) photographic album of man-made and naturally occurring materials. What is interesting is that only a handful of features have been proposed as candidate attributes, as opposed to the high number obtained by enumerating sensory channels or cell types. Implied is a suggestion that not only are higher order features $u$ seful in judgments of complex textures, but that the number of useful features is small. For example, Tamura, Mori, and Yamawaki (1978) studied paired comparisons of textures according to six textural attributes (i.e., coarseness, contrast, directionality, line-likeness, regularity, and roughness). The emphasis, however, was on developing computational analogues of the attributes and not on whether the attributes were those actually used in everyday untutored judgments of texture. The study and attributes of Amadasun and King (1989) were similar (coarseness, contrast, busyness, complexity, and texture strength). Both studies were only moderately successful in using computational approximations of textural attributes for the prediction of perceived overall texture similarity.

Rao and Lohse $(1993,1996)$ recognized the need to investigate attributes that are relevant to actual judgments of natural textures. Comparing ratings of the Brodatz (1966) textures along attribute scales with the results of a later sorting task in which no explicit criteria were specified, they found that these high-order judgments could be embodied in relatively low-dimensional representations. They proposed that three dimensions were important to texture perception - namely, repetitive, contrast/ directional, and granular/coarse/low-complexity. Many of their findings are consistent with our independent study; however, we also try to resolve two major issues: How idiosyncratic is texture perception (intersubject variability), and does statistical testing reveal texture space to be homogeneous?

We examine six attributes as candidate descriptors of texture space: lightness, contrast, edginess, coarseness, directionality, and regularity. Texture lightness, a feature not previously used in the texture literature, seemed a fairly obvious choice, since it has a physical correlate in mean surface reflectance and should be of importance in comparisons of most monochrome texture pairs; most of our other attributes have found prior use in the literature (e.g., Tamura et al., 1978). Whereas color lightness varies with reflectance from point to point in a texture, texture lightness involves a judgment about the spatial average of reflectance - that is, whether the texture surface as a whole is generally light or dark. Unfortunately, earlier perceptual studies of the Brodatz textures neglected to explicitly exclude lightness as a confounding factor, either by manipulation of the stimuli or by specific instructions to the observers. We have found that a few of our candidate attributes, including lightness and, in particular, coarseness and regularity, account for the greater part of the variance in rapid untutored or gestalt-type judgments of the overall dissimilarity of texture pairs. Our results suggest that, despite the rich early neural representation, the practical differentiation of textured materials is based on a relatively low number of dimensions, perhaps four or five, and that our six attributes are useful directions in that space, despite nonorthogonality and other complexities. We find that rapid untutored judgments of natural textures is an experience invariant across observers and that texture space is relatively homogeneous. Also, texture judgments seem to access a common representation, even when viewed under impoverished viewing conditions or when different types of judgments are asked of the observers.

There are a number of features unique to our study. (1) To avoid possible bias from prior knowledge of texture attributes, we took the simple step of first requesting ratings of the inherent overall dissimilarity of pairs of textures; the candidate attributes were not revealed to the subjects until the second part of the session, when ratings of the specific dissimilarity of the texture pairs were requested for each of the six attributes in turn. (2) We examined texture lightness as a candidate attribute and found it to be a significant predictor of overall dissimilarity. (3) We found a dimensionality of around four, slightly higher than previous estimates, which may be due to our inclusion of lightness as an attribute. (4) Our explicit treatment of intersubject variability found good agreement between observers. (5) We performed supplementary experiments, using a wide range of viewing distances and light levels. (6) We replicated part of our study for errors at an identification task, with essentially similar conclusions about dimensionality; this suggests the possibility that low dimensionality is not restricted to tasks that involve coarse judgments but is typical of texture perception as a whole.

\section{METHOD}

\section{Stimuli}

Sixty samples were cut from the Brodatz (1966) album of halftone prints of textures (Appendix A). The Brodatz set approximates 
the textural variety in man-made and naturally occurring materials and provides convenient opportunity for comparison of our results with those from the prior literature. This set has received intensive study in both human and computer texture vision. An attempt was made to maximize the number and diversity of texture samples. For ease of presentation and comparisons of textures with themselves, nonidentical samples of the textures with the same Brodatz numbers were cut from another copy of the Brodatz book, for a total of 120 samples. The textures were divided into five sets of 12 patterns to avoid prohibitive numbers of texture pairings and to allow a crossvalidation between groups of textures (see the Variability Across Texture Sets and Observers section). Each texture sample was $10 \times$ $10 \mathrm{~cm}$, subtended $6.4^{\circ}$ of visual angle, and was mounted on an $18 \times$ $18 \mathrm{~cm}$ piece of gray matte board with a reflectance of 0.30 . The mean reflectance of the Brodatz textures was 0.25 . Pairs of textures were presented with the side borders adjacent to each other so that the angular subtense between the centers of the texture pairs was $11.4^{\circ}$. The viewing distance was $90 \mathrm{~cm}$. The lighting was northern daylight, supplemented by fluorescent light. The luminance of the gray borders of the prints was $37.5 \mathrm{~cd} / \mathrm{m}^{2}(S D=8.9)$, giving a retinal illuminance, for a measured mean pupil diameter of $2.5 \mathrm{~mm}$, of 94.8 $\mathrm{Td}$ for the border.

\section{Subjects}

Twelve observers ( 6 male, 6 female) participated in the main experiment. All the observers had normal or corrected-to-normal visual acuity. Six had previous experience with texture judgments in pilot experiments. The other 6 observers were naive as to the specific aims of the experiment.

\section{Ratings of Overall Dissimilarity $(\Delta)$}

The observers spent $1-2$ min familiarizing themselves with the textures by viewing five $21.6 \times 27.9 \mathrm{~cm}$ panels held in hand, each panel providing small samples of the 12 textures. The observers were then asked to make two types of judgments. The first half-session required judging overall dissimilarity, with the criteria for judgment left to the observer's discretion (the symbol $\Delta$ will frequently be used to denote overall dissimilarity judgments). The 7-point scale ranged from 1 (very similar pairs) to 7 (very dissimilar pairs). The $\Delta$ ratings were completed before the instructions for the second half-session were explained, so as to avoid bias.

\section{Ratings of Attribute Dissimilarity $\left(\Delta^{A}\right)$}

The second type of judgment involved ratings of dissimilarity with respect to a set of six candidate attributes ( $\Delta^{A}$ ratings), one at a time. The process of selecting the candidate attributes included a review of the literature on natural texture vision (e.g., Bhushan, Rao, \& Lohse, 1997; Tamura et al., 1978), as well as a dictionary and thesaurus search to consider new attributes and to guide our choices between synonyms. The number of attributes was limited to six. Although this admittedly does not offer an exhaustive search, we thought that our particular choices offered a relevant and varying array of possibilities, while being a practicable number for our experimental paradigm. We included the novel attribute of texture lightness: Mean surface reflectance is an invariant property that is integral to a texture's definition, ' and we thought that its subjective analogue might have perceptual relevance. In our analyses, we examined whether the candidates were well chosen. The attributes were defined for the observers as follows. (1) Texture lightness ${ }^{2}$ (1) was defined as an assessment of the average perceived brightness from all parts of the texture plate. (2) Texture contrast $^{3}$ (c) was defined as a measure of the degree of variation of light levels within each texture. For example, a plate without much contrast has a relatively uniform reflectance. (3) Edginess (e) was a measure of edge definition within the texture. Edginess may be thought of as the op- posite of fuzziness. (4) Coarseness (f) of textures can vary in two different ways, in keeping with the definition of Tamura et al. (1978). If texture is represented as a repeated placement of fundamental elements, one texture will be coarser than another if either the elements or their spacings are larger than those of the other. A texture that is not coarse may be thought of as fine. (5) Directionality (d) was defined by both its element shape and a placement rule (again, in agreement with Tamura et al., 1978). Directionality could exist for more than one orientational axis, but a texture that was uniformly directional for only one axis was assessed as having the highest directionality. (6) Regularity (r) assesses order within a texture. It is the antonym of randomness.

The observer's task was to rate each texture pair according to each of the above attributes on an integer scale. A coarser rating scale facilitated rapid, robust judgments. The observers were instructed to assign a rating of -2 if the texture on the left was deemed to have much more of the assessed attribute than the one on the right, and a rating of +2 for the converse case. If there was little or no difference between the textures, the observers were to assign a rating of $0 ;+1$ or -1 was to be assigned to intermediate judgments.

\section{Stimulus Presentation and Data Collection}

The number of presentations on each side was balanced to ensure that each texture was repeated at relatively well spaced intervals (Ross, 1934). Viewing times were not limited, but most comparisons took approximately $5 \mathrm{sec}$ to complete. For the $\Delta$ ratings, all 78 possible pairs (including self-comparisons) of a set of 12 textures were presented to each of the 12 observers. The total number of $\Delta$ ratings was 4,680 (12 observers/pairing $\times 78$ texture pairings $\times 5$ texture sets). For the $\Delta^{A}$ ratings, the number of attributes made it impractical for each observer to exhaustively complete all the pairings. Instead, pooling across observers provided four replications for each texture pair. The total number of $\Delta^{A}$ ratings amounted to 9,360 (6 attributes $\times 4$ observers/pairing $\times 78$ texture pairings $\times$ 5 sets). A full session for 1 observer lasted about $2.5 \mathrm{~h}$. The analyses pooled ratings across observers, except where specified.

\section{Analyses}

Multidimensional scaling. Individual texture samples were viewed as points populating a multidimensional space where the distances between the points matched, as closely as possible, the experimental $\Delta$ ratings for overall or innate dissimilarity (for illustrations of texture space, see Rao \& Lohse, 1996, Figure 4). In a well-matched configuration, pairs of textures that elicit larger $\Delta$ ratings (i.e., look more dissimilar) will tend to be situated farther apart in the space, and those with lesser ratings, closer together. To discover the correct dimensionality, we employed a nonmetric multidimensional scaling (MDS) algorithm (Kruskall, 1964), which attempted to match the ordinal values of the $\Delta$ ratings for texture pairs with the respective distances between the texture pairs in the MDS space. In a perfect match, MDS distances between pairs of textures would be a monotonically increasing function of the $\Delta$ ratings. When there are no a priori grounds for assuming a particular relationship between the stimuli, a Euclidean metric is commonly employed and was used in all the MDS analyses. To assess the goodness of fit, we used stress (Kruskall \& Wish, 1978), which ranged from 0 to 1 for good to bad fits, respectively. We also provide the accompanying $R^{2} \mathrm{~s}$, which give the proportion of variance in the $\Delta$ ratings accounted for by the corresponding MDS distances. Common practice is to increase the dimensionality until stress is less than 0.10 . After estimating dimensionality, the experimenter can then search for meaningful axes in the MDS representation of perceptual space (we will denote this by $M D S-\Delta$ space). To avoid difficulties in visually inspecting MDS- $\Delta$ solutions with dimensions higher than two, we used independently derived MDS scales (based on indi- 
vidual $\Delta^{A}$ attribute ratings) that placed the textures along a 1-D MDS$\Delta^{A}$ scale defined by an attribute $i(i=1-6)$ that had possible relevance to the judgments of overall dissimilarity $(\Delta)$. After regressing the MDS- $\Delta^{A}$ values of the texture set against the MDS- $\Delta$ coordinates, the relevance and orientation of scale $i$ in the texture space (MDS- $\Delta$ ) could be ascertained from the standard $R^{2}$ and $\beta$ coefficients, respectively.

Multiple linear regression. The aim of this analysis was to predict the behavior of a dependent variable, using a weighted sum of independent predictor variables. In our case, we attempted to predict the $\Delta$ ratings by using the $\Delta^{A}$ ratings as predictor variables and the squared correlation coefficient $R^{2}$ as the measure of goodness of fit.

Cross-validation. The disadvantage of the usual method of regression analysis is that it assesses the fit of the model to the data but does not accurately predict the model's performance when confronted with new data. Also, intermediate values of $R^{2}$ are often difficult to interpret. Cross-validation addresses both these concerns by making predictions of new data in the actual units of the predicted variable. Another concern is that correlated predictor variables can cause instability in the coefficients of the regression equation, which may lead to poor predictions of new data. Although crossvalidation does not remedy this, it can provide a measure of the severity of this difficulty. In our case, the technique assessed a regression model's predictive power, using the difference between predicted $\Delta$ ratings and actual $\Delta$ ratings as an estimate of the prediction error. First, the data were partitioned into a training set and a test set. The regression coefficients were obtained, using the $\Delta$ and $\Delta^{A}$ values from the training set. Then, the $\Delta^{A}$ values of the test set, together with the training set regression coefficients, were used to predict the test $\Delta$ values. The average unsigned difference between these predicted $\Delta$ values and the actual test-set $\Delta$ values, taken over all possible training and test sets, was the estimate of prediction error. In practice, the data were split into $K$ equal parts, the $k$ th part being taken as the test set and the prediction error being determined by using the remaining $K-1$ parts as training data. Performing this for each of $k=1,2, \ldots, K$ parts, one could combine the $K$ estimates of prediction error (Efron \& Tibshirani, 1993). The whole procedure was then repeated for models with varying numbers of dimensions.

Redundancy analysis. This provides a measure of the amount of variance in one set of variables explained by another (Stewart \& Love, 1968). One of our interests was assessing variability between observers' ratings and, specifically, the $\Delta$ ratings, since these represented everyday, untutored judgments of natural textures. Separate MDS- $\Delta$ solutions were derived for each individual's $\Delta$ ratings. Then, for instance, Observer A's coordinates were employed as predictor variables for those of Bs. With perfect prediction, the 2 observers would have identical MDS spaces. The technique is equivalent to regressing, in turn, each of the variables (MDS- $\Delta$ coordinates) of the first set over all the variables of the second set and averaging the resulting multiple correlation coefficients. More explicitly, the technique produces a redundancy coefficient $\overline{R^{2}}$, which is an average of the proportion of explained variance $\left(R^{2}\right)$ that is accounted for by a particular canonical variate, each $R^{2}$ being weighted by its associated squared canonical correlation (Stewart \& Love, 1968). The redundancy coefficient $\overline{R^{2}}$ can be regarded as an average multiple$R^{2}$ (of linear regression analysis).

\section{Impoverished Viewing Conditions and an Identification Task}

We explored the effects of systematic decreases in illuminance $(99.3,17.7,3.14,0.56,0.10 \mathrm{Td})$ or increases in viewing distance $(0.9,8.2,15.5,22.9,30.2 \mathrm{~m}$ at mean illuminance of $902 \mathrm{Td}$ for Brodatz texture $\mathrm{D} 34$, which had reflectance $=0.61$ ) on the $\Delta$ and $\Delta^{A}$ ratings, using 10 subjects in each case. Bendzsack (1996) and Nikmanesh (1996) subsequently replicated the two studies in full on two new groups of 10 subjects and Texture Set 3 (Appendix A) with almost the same results.

We also examined observers' performances on a task involving the identification of 20 different texture samples with varying distance, to ascertain whether errors in identification were inversely related to ratings of dissimilarity. Ten sets of 20 different $12 \times$ $12 \mathrm{~cm}$ Brodatz texture samples were mounted in randomly ordered $4 \times 5$ arrays on 10 gray panels with a reflectance of 0.30 . In addition to all 12 textures of Set 1 , Appendix A, the following were included: D4 (pressed cork), D12 (bark of tree), D16 (herringbone weave), D37 (water), D55 (straw matting), D76 (oriental grass fiber cloth), D86 (ceiling tile), and D109 (handmade paper). The five distances were as given above. The subjects provided their best guesses at identifying the distant samples, referring to a small panel of all 20 textures (each $3 \times 3 \mathrm{~cm}$ of a Brodatz plate) held in the hand to avoid any confusion over names.

An MDS analysis was performed, using transformed pairederror rates as the dissimilarity input to the MDS algorithm. The transformation into dissimilarities assumes an inverse proportionality of the confusion between a pair of textures and the dissimilarity between them. For each distance, the dissimilarity matrix was derived as follows: The stimulus/response matrix $(\mathbf{M})$ was added to its own transpose, resulting in a symmetric matrix-say, $\mathbf{A}(\mathbf{A}=\mathbf{M}+$ $\mathbf{M}^{\mathrm{T}}$ ). The dissimilarity matrix (D) was then calculated by subtracting $\mathbf{A}$ from a matrix composed solely of elements whose values equaled that of the highest element of $\mathbf{A}$ ( $k$, a scalar). So, in algebraic notation, $\mathbf{D}=k 1 \underline{1}^{\mathrm{T}}-\mathbf{A}\left(\underline{11}^{\mathrm{T}}\right.$ is matrix with $1 \mathrm{~s}$ as elements $)$. For an alternate method, see Getty, Swets, Swets, and David (1979).

\section{RESULTS}

We employed regression analysis and MDS to ascertain the dimensionality of the overall dissimilarity judgments $(\Delta)$ and to evaluate the relevance of the attribute dissimilarity ratings $\left(\Delta^{A}\right)$ to the judgments. It should be noted that a city-block metric is implied whenever raw $\Delta$ and $\Delta^{A}$ rating values are used and a Euclidean metric when dealing with MDS estimates of the spaces that represent the $\Delta$ and $\Delta^{A}$ data as distances. However, any differences between the metrics are probably obscured by the noise in our data. We then used cross-validation and redundancy analysis to check that our analyses of texture space had general utility across observers and texture sets. To better characterize the attributes themselves, MDS analyses and correlations were next performed on the $\Delta^{A}$ judgments. Finally, we examined how resistant the implied perceptual representation of textures was to major changes in the task or viewing conditions.

\section{The Dimensionality of Overall Dissimilarity ( $\Delta$ Ratings)}

MDS analysis provided evidence for a low dimensionality in the overall dissimilarity judgments $(\Delta)$.

Separate stress values for dimensionalities varying from one to four were determined for the mean observer $\Delta$ ratings of each of the five texture sets. The averaged stresses are summarized in Figure 1. Stress was .31 at one dimension, declining to .07 by three dimensions; associated $R^{2}$ values were .68 and .96 , respectively. Four-dimensional (4-D) solutions showed some improvement (stress $=.03$, $R^{2}=.99$ ), but must be viewed with caution because of the 


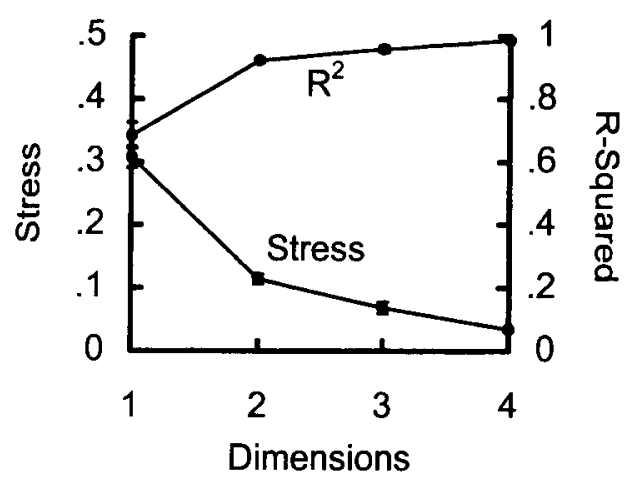

Figure 1. Main experiment: Overall dissimilarity judgments $(\Delta)$ for dimensionalities of the implied perceptual space. Stress values, averaged over the five texture sets, are shown for analyses of increasing dimensionality. A dimensionality of three or four is suggested with stress values of $.07\left(R^{2}=.96\right)$ and $.03\left(R^{2}=.99\right)$, respectively. Viewing distance, $0.9 \mathrm{~m}$; mean illuminance for gray borders of prints, $94.8 \mathrm{Td}$.

possibility of overfitting (with 12 textures per set). The implied texture space, derived from MDS analyses of $\Delta$ ratings, was at minimum 3-D and, probably, at least 4-D; this conclusion was supported by the results presented in the following sections. We will subsequently refer to this space as MDS- $\Delta$ space.

\section{Attribute Dissimilarities $\Delta^{A}$ as Predictors of Overall Dissimilarity $\Delta$}

Regression analyses using $\Delta$ versus $\Delta^{A}$ data. We explored the dependency of dissimilarity judgments $\Delta$ on candidate attribute dissimilarity judgments $\Delta^{A}$, using multiple linear regression. We attempted to predict overall dissimilarity $(\Delta)$ judgments, using the attribute judgments $\left(\Delta^{A}\right)$ as predictor variables.

To obtain the optimal model for any given number of predictors, an exhaustive survey of all 63 possible regression equations was undertaken (Figure 2). Each point in Figure 2 represents the proportion of variance (multiple $R^{2}$ ) in the $\Delta$ judgments accounted for by a regression model that included the attribute dissimilarities as labeled in order of decreasing importance. For example, the four attributes predicting the point (arrowed) are $f$ (coarseness), $r$ (regularity), $d$ (directionality), and I (lightness). With four or five parameters, the best regression models (the top points in each column of points) attained multiple $R^{2}$ values exceeding .70, and little was gained by adding a sixth parameter. Filled circles show that the two best overall predictors are coarseness (f) and regularity (r), although all the attributes had some use. Significant but lesser predictors for the main viewing condition $(94.8 \mathrm{Td}$, 90-cm distance) were lightness, directionality, contrast, and edginess.

Regression analysis using multidimensional scaling transformations of the $\Delta$ and $\Delta^{A}$ ratings. We evaluated the proportion of variance in individual MDS- $\Delta^{A}$ scales accounted for by the MDS- $\Delta$ space coordinates (Shiffman, Reynolds, \& Young, 1981). First, 6 one-dimensional MDS scales were constructed from the dissimilarity ratings for the six individual $\Delta^{A}$ attributes; each texture was a point on such a scale. Each texture also held 3-D or 4-D coordinates already obtained from the MDS- $\Delta$ estimate of texture space, given in the Dimensionality of Overall Dissimilarity ( $\Delta$ Ratings) section, above. So, one can regress the six 1-D MDS- $\Delta^{A}$-derived coordinates (one attribute at a time) against the 3- or 4-D MDS-derived coordinates as predictors. This optimally orients each attribute axis in the MDS- $\Delta$ space, and the goodness of fit of the MDS$\Delta^{A}$ axis to the MDS- $\Delta$ space would be shown by $R^{2}$ (Shiffman et al., 1981). For example, if coarseness was relevant to observers' $\Delta$ judgments of textures, the textures should vary according to coarseness along a particular direction in the MDS- $\Delta$ space, and the resulting $R^{2}$ should be high if the 1-D MDS- $\Delta^{A}$ coarseness scale faithfully captures the variance along this direction in MDS- $\Delta$ space. It might be noted that these $R^{2} \mathrm{~s}$ can be elevated in comparison to the $\Delta$ versus $\Delta^{A}$ regressions (Results 2.1) since MDS coordinates are used as predictors, rather than raw ratings.

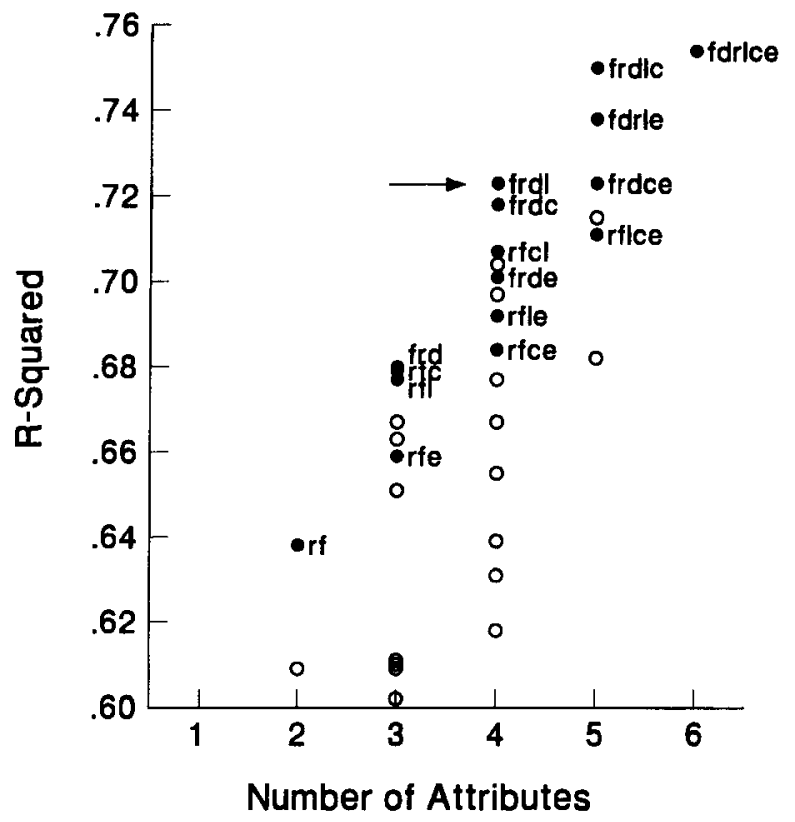

Figure 2. $\Delta$ versus $\Delta^{A}$ relationship in an exhaustive survey of the 63 possible attribute combinations. Each point represents the propertion of variance (multiple $R^{2}$ ) in the overall dissimilarity $(\Delta)$ judgments accounted for by a regression model that includes the attributes as labeled (abbreviated) in order of decreasing importance. The $\boldsymbol{x}$-axis indicates the number of attribute dissimilarities ( $\Delta^{A}$ entered in the respective equations). The best of the six possible one-predictor models (not visible) just failed to exceed $R^{2}=.5$. Of the 15 two-predictor models, the one using dissimilarities of coarseness ( $($ ) and regularity ( $r$ ) accounts for .64 of the variance in the $\Delta$ judgments. By four or five parameters, the best regression models attained $R^{2}$ s exceeding .70 . The sixpredictor model offered no real improvement. The two best predictors overall were dissimilarities in coarseness (f) and regularity ( $r$ ) (filled circles denote models including $f$ and $r$ ). Lesser predictors are lightness (l), directionality (d), contrast (c), and edginess (e). 
Table 1

Regressions of Attribute Scales on Multidimensional Scaling (MDS) Coordinates $\left(R^{2}\right)$

\begin{tabular}{lcc}
\hline \multicolumn{1}{c}{ Attribute } & $3-\mathrm{D}$ & $4-\mathrm{D}$ \\
\hline Coarseness & .85 & .91 \\
Regularity & .87 & .90 \\
Directionality & .87 & .90 \\
Contrast & .54 & .70 \\
Lightness & .48 & .66 \\
Edginess & .64 & .72 \\
\hline
\end{tabular}

Note--Linearity of MDS space: One-dimensional (1-D) MDS scales of the textures were constructed from the ratings for each individual $\Delta^{A}$ attribute. The 1-D scales were then regressed over the corresponding three-dimensional (3-D) and four-dimensional (4-D) MDS spaces derived from $\Delta$ ratings of the same textures. In the 3-D solutions, coarseness, regularity, and directionality show impressive $R^{2} \mathrm{~s}$, suggesting that any of these attributes could serve as axes in a low-dimensional texture space. The $R^{2} \mathrm{~s}$ for these attributes show only modest improvement for 4-D solutions. In proceeding to the 4-D solutions, contrast, lightness, and edginess demonstrate notable increases in $R^{2} \mathrm{~s}$. All candidate attributes are satisfactory linear directions in 4-D MDS- $\Delta$ space.

For the 3-D configurations, coarseness, regularity, and directionality demonstrated high $R^{2} \mathrm{~s}$ (around .87; see Table 1). In addition, in the 4-D solutions, contrast, lightness, and edginess attained $R^{2} \mathrm{~s}$ of approximately .70, implying that all six candidate attributes were satisfactory linear directions in a 4-D MDS- $\Delta$ estimate of texture space.

\section{Variability Across Texture Sets and Observers}

One possibly serious concern about the literature and the above analyses is the use of seemingly very subjective quick judgments. We explore this issue in the next two sections with cross-validation methods (Efron \& Tibshirani, 1993). Another problem is that a fixed body of data is generally better fitted by more parameters. This is not the case in cross-validation, where introducing irrelevant parameters (dimensions) will eventually worsen the fit of new data (for an older analytic approach, see Schwartz, 1978). We partitioned the data set and developed the model (multiple linear regression equation) on one portion (training set), estimating the prediction error on the remaining data (test set) and pooling the estimated prediction errors (see the Method section).

For our data, the regression coefficients (the weightings for the $\Delta^{A}$ judgments) from the training sets were used to predict new $\Delta$ ratings, given the $\Delta^{A}$ ratings of the remaining (test) data. The average absolute value of the difference between the predicted $\Delta$ ratings and the actual $\Delta$ ratings of the test set was the prediction error. Prediction errors are in $\Delta$ rating units, ranging from 0 to 6 , since actual $\Delta$ judgments range from 1 to 7 . Prediction error was expected to show a limited reduction with an increase in the number of parameters, an excessive number of parameters causing no improvement or even a worsening of the prediction. We find below that useful regression models use four attributes.

Cross-validation for texture sets. A real concern was the possibility that texture discrimination is considerably texture-set dependent. We calculated the average prediction error, using cross-validation, rotating the texture set that served as test set (see the Method section). The results, shown in Figure 3, confirmed that the regression models were applicable across texture sets. With only one attribute, the predictive power of the models was poor, with errors of around 2.0 rating units. With four attributes the error decreased to only 0.9 rating units on a scale of $1-7$. Considering that the dissimilarity judgments $(\Delta)$ of wholly new sets of textures were being predicted, this result supports the reliability of dissimilarity judgments $\left(\Delta, \Delta^{A}\right)$ and low-dimensional models.

Cross-validation for observers. Intersubject variability is a major concern when positing a stable, unified texture space common to all observers. So far, our analyses have pooled data across all observers and have suggested that, for the observers as a group, there were robust regularities in the judgments of texture pairs. However, do rapid untutored judgments (ratings) by 1 observer have much relevance to another's? Figure 4 shows intersubject variability measured by cross-validation prediction error. There were four repeats of each $\Delta^{A}$ rating, so each point was an average of four calculations of prediction error. Since we predicted 1 observer's $\Delta$ ratings by using regression models trained on 3 other observers' ratings, we obtained a useful measure of intersubject variability. With one predictor, there was already a useful prediction of the remaining observers' ratings. With four attributes as predictors, prediction error was around 1.2 rating units on a scale of $1-7$. Thus, $\Delta$ ratings were similar across observers.

Redundancy across observers. It is likely that the cross-validation prediction errors were inflated by the imperfect $\Delta-\Delta^{A}$ linear regression models used in making the predictions. To assess the similarity of observers' perceptual spaces more directly, redundancy analysis was employed (Stewart \& Love, 1968). We gained the advantage of excluding the $\Delta^{A}$ attributes - they may have been

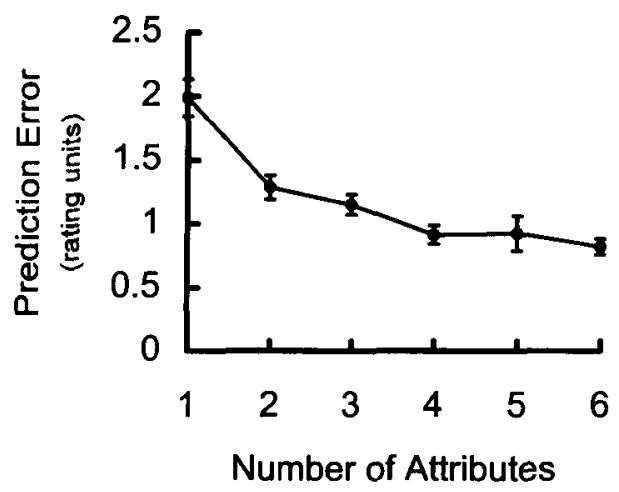

Figure 3. $\Delta$ vs. $\Delta^{A}$ relationship: cross-validation across texture sets. Prediction error was used as a measure of across-set variability in perceived overall dissimilarity. Shown are the average prediction errors for models with increasing numbers of attributes. This confirms that the regression models for dissimilarities were applicable across different sets of textures. With one attribute, the predictive power of the models was poor, with errors of around 2.0 rating units. With four predictor models, the error decreased to 0.9 , suggesting that at least four attributes are required to account for the variance in the $\Delta$ judgments. 


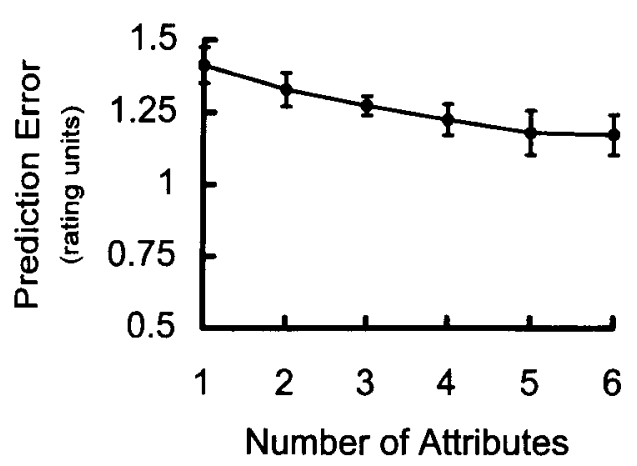

Figure 4. $\Delta$ vs. $\Delta^{A}$ relationship: cross-validation across observers. Prediction error was used as a measure of the intersubject variability in perceived overall dissimilarities $(\Delta)$. Shown are the average prediction errors for models with increasing numbers of attributes. Cross-validation is explained in the Method section. Since, in this case, we predicted 1 observer's $\Delta$ ratings by using regression models trained on other subjects' $\Delta$ and $\Delta^{A}$ values, we measured intersubject variability in rating units. Notice that, with one predictor, there was already reasonable prediction of another subject's $\Delta$ ratings. There was a steady decrease in prediction error, with average errors of around 1.2 for models with five attributes.

imperfect descriptors for texture judgments-and examining only the $\Delta$ judgments. A given texture has one set of coordinates in the MDS- $\Delta$ space of one observer, and another set for another observer. The technique is equivalent to regressing, in turn, each of the variables (MDS coordinates) of the first set over all the variables of the second set and averaging the resulting squared multiple correlation coefficients. The redundancy coefficient $\overline{R^{2}}$ can be regarded as an average multiple- $R^{2}$ (of linear regression analysis).

For each of the five sets of textures, the $\overline{R^{2}}$ s were determined for all observer combinations. Table 2 shows computations for the 3-D and 4-D MDS configurations that provided the most reasonable fits to the $\Delta$ data. The average over all the texture sets of the $4-\mathrm{D} \overline{R^{2}}$ s was .63 . For 3-D MDS configurations, the $R^{2} \mathrm{~s}$ were slightly lower. We concluded that there were good matches between observers' perceptual spaces, with the 4-D configurations being better than the 3-D ones. This approach provided a separate measure of intersubject variability from the $\Delta-\Delta^{A}$ model cross-validation in the Cross-Validation for Observers section, above, and yielded the same conclusion. Direct correlations between different observers' raw $\Delta$ ratings averaged to $R^{2}=.61$.

\section{Nature of the Attributes}

Correlations between attributes $\left(\Delta^{A}\right)$. Although all the $\Delta^{A}$ attributes may serve as useful axes (directions) in a texture space, they need not be mutually orthogonal or cardinal. There are a priori grounds for suspecting that a number of the attributes are interrelated. For example, Tamura et al. (1978) noted that the sharpness of edges (edginess) and the period of repeating patterns (coarseness) may affect judgments of contrast (contrast).
Correlations were determined for all possible pairs of attributes (Table 3 ). The highest correlations were between regularity and directionality $(r=.72)$ and between contrast and edginess $(r=.64)$. Lower correlations were obtained between contrast and coarseness $(r=.39)$, edginess and regularity $(r=.41)$, and coarseness and edginess $(r=.45)$. These pairs, then, cannot be strictly orthogonal dimensions in a texture space. This pattern of correlations also explains how each of the six 1-D attribute scales could attain such high $R^{2} \mathrm{~s}$ when regressed with the corresponding 4-D MDS configurations (see the Redundancy Across Observers section) - six scales could only serve as meaningful axes in a 4-D MDS space if they were not all mutually orthogonal.

Dimensionality of the individual attributes $\left(\Delta^{A}\right)$. The $\Delta^{A}$ judgments have thus far been assumed to be truly unidimensional attribute scales. There are good reasons to suspect, however, that the $\Delta^{A}$ judgments themselves may be multidimensional. For instance, Tamura et al. (1978) suggested that four factors contribute toward contrast judgments - namely, the dynamic range and polarity of the gray-level distribution, the sharpness of edges, and the period of repeating patterns. It remains a possibility, then, that the variance in $\Delta^{A}$ judgments for contrast might be captured by a 4-D contrast space. Coarseness may deserve a two-dimensional (2-D) space, since both the size and the space of repeating elements figure into judgments. Similar arguments could be made for the other attributes, except, perhaps, texture lightness. In the latter case, one might hope for a unidimensional representation, because spatially averaged reflectance is a single physical variable; however, it is not entirely clear how receptive field signals are combined to estimate this physical quantity. Differences in mean physical reflectances between the texture samples are highly correlated with corresponding assessments of texture lightness $(r=.88)$.

To explore the possible multidimensionality of attributes, MDS analyses were performed on the $\Delta^{A}$ ratings of each separate attribute (Figure 5). None of the attributes attained stress levels below 10 with only $1-D$ solutions, so there is the question of whether any of the attributes, including lightness, are simple scalars. Lightness, regularity, and directionality all attain stresses below .10 by two dimensions. Edginess, coarseness, and contrast require two or three dimensions. These results suggest that, if mea-

Table 2

Between-Observer Redundancy Coefficients

\begin{tabular}{lccc}
\hline Dimensionality & Average & $S D$ & Range \\
\hline Three-dimensional & .54 & .11 & $.21-.88$ \\
Four-dimensional & .63 & .11 & $.28-.88$ \\
\hline
\end{tabular}

Note-Between-observer redundancy coefficients, averaged over subjects and texture sets. As a measure of intersubject variability, the variances shared between individual observers' MDS- $\Delta$ configurations were determined, using redundancy analysis. The table summarizes the redundancy coefficients $\left(\overrightarrow{R^{2}}\right)$, averaged across the 66 possible pairings of 12 subjects and the five texture sets. Matches between observers' perceptual spaces are reasonable to very good, the four-dimensional configurations being the better. 
Table 3

Correlations Between Attributes (r)

\begin{tabular}{crrrrr}
\hline Attribute & $\mathrm{l}$ & $\mathrm{r}$ & $\mathrm{d}$ & $\mathrm{c}$ & $\mathrm{f}$ \\
\hline $\mathrm{r}$ & -.03 & & & & \\
$\mathrm{~d}$ & -.06 & $.72^{*}$ & & & \\
$\mathrm{c}$ & .20 & -.06 & -.08 & & \\
$\mathrm{f}$ & -.03 & -.09 & -.18 & $.39^{\dagger}$ & \\
$\mathrm{e}$ & .16 & $.41^{\dagger}$ & .27 & $.64^{*}$ & $.45^{\dagger}$ \\
\hline
\end{tabular}

Note- $\Delta^{A}$ ratings: correlations between attributes. Shown are the correlations between all the possible pairings of $\Delta^{A}$ ratings for each set and pooling across sets. Attribute abbreviations: 1, lightness; c, contrast; e, edginess; $f$, coarseness; $d$, directionality; and $r$, regularity. The superscripts mark notable correlations. The highest correlations include the pairs r:d and c:e $\left({ }^{*}\right)$; lesser correlations include $\mathrm{c}: \mathrm{f}, \mathrm{e}: \mathrm{r}$, and $\mathrm{f:e}(\dagger)$. These pairs, then, cannot be strictly orthogonal dimensions in a texture space.

surement noise can be ignored, the individual attributes are not scalars - that is, are not infinitesimally narrow 1-D axes in texture space.

\section{Judgments Under \\ Impoverished Viewing Conditions}

Since the overall dissimilarity judgments are relatively stable across different observers and sets of textures, it can be asked whether they remain constant when the stimuli are presented under impoverished viewing conditions. In supplementary experiments, observers were asked to make the same types of judgment of texture pairs ( $\Delta$ and $\Delta^{A}$ ratings), presented under low illumination or greater viewing distances (see the Method section).

The dimensionality of overall dissimilarity $(\Delta)$. At the most general level, reducing stimulus visibility did not alter the dimensionality of the perceptual spaces derived from MDS- $\Delta$ analyses (Figure 6). For the varying illuminance condition, all illuminance conditions showed satisfactory stress values of below .10 for the 4-D configurations; changing the viewing distance produced similar results. These results were not significantly different from those of the main experiment, which used a short viewing distance and high illuminance (Figure 1; $95 \mathrm{Td}$, $0.9 \mathrm{~m}$ ). However, the stress curves (Figure 6, unfilled circles, top and bottom panels) were a little lower for the extreme viewing conditions (condition 5:0.1 Td or $30 \mathrm{~m}$ ), suggesting a lower dimensionality, as might, perhaps, be expected.

Relevant attributes: $\Delta$ versus $\Delta^{A}$ relationship. To examine which attributes were significant, exhaustive regressions were performed with $\Delta$ ratings being predicted by the $\Delta^{A}$ ratings, in the manner of Figure 2 . If we focus on the topmost points in Figure 7 for each attribute number $(x$-axis), we see that all the illuminance levels demonstrated high maximum $R^{2}$ s that increase with added attributes and reached a plateau at around $R^{2}=.80$ (arrows) for four attributes. Although the $R^{2} \mathrm{~s}$ for the four highest illuminances followed a similar pattern, the early plateau of the maximum $R^{2} \mathrm{~s}$ at the lowest illuminance denotes a sharp reduction in the number of useful attributes to one or two at that illuminance.
With increases in viewing distance, there was a more gradual change in the distributions of maximum $R^{2} \mathrm{~s}$ (topmost points). Again, at each distance, maximum $R^{2} \mathrm{~s}$ increased with added attributes and, in this case, reached a plateau at around . 70 for 3-4 attributes. At the furthest distance, however, $R^{2}$ was reduced, and there was, effectively, only one viable attribute. Closer scrutiny of the regression equations, in either of the impoverished conditions, shows that texture lightness explains most of the variance. Even under the best viewing conditions (highest illuminance or closest distance), lightness had more significance as a predictor in the supplementary experiments than in the main experiment at high illuminance and short distance. The wide range in viewing conditions may have increased the salience of lightness as an attribute in the supplementary experiments. Most ( $80 \%)$ of the observers began the distance experiment at a distance considerably greater than the $0.9 \mathrm{~m}$ of the main experiment; lightness was also a prominent attribute in the illuminance experiment, in which retinal illuminance was usually more than $1 \mathrm{log}$ dimmer than that in the main experiment. Still, coarseness and regularity, which were the major attributes in the main experiment, figured again as important predictors of overall dissimilarity judgments $(\Delta)$ for the first four illuminances $(99.6-0.56 \mathrm{Td})$ and the first three distances $(0.9-$ $15.5 \mathrm{~m})$.

Direct comparisons of $\Delta$ ratings. As a more stringent measure of the invariance of judgments, one could make direct comparisons of the $\Delta$ ratings under impoverished and optimal (highest illuminance or closest distance) conditions. Figure 8 (squares) shows Pearson correlations between $\Delta$ ratings at the closest distance $(0.9 \mathrm{~m})$ and comparable ratings made at the further distances $(8.2-30.2 \mathrm{~m})$.

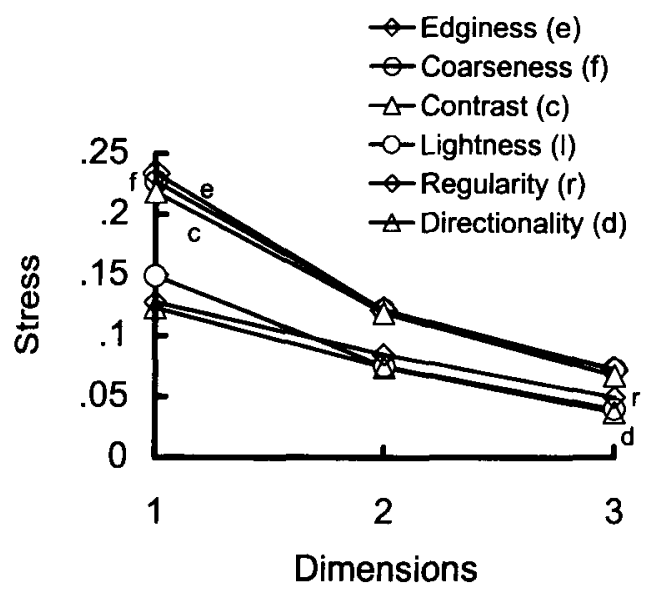

Figure 5. Dimensionality of the individual attributes. Shown are the stress values for multidimensional scaling (MDS) analyses of the $\Delta^{A}$ ratings of each separate attribute. None of the attributes attain stresses below $\mathbf{. 1 0}$ with only one MDS dimension. Lightness, regularity, and directionality all attain stresses below .10 by two dimensions. Edginess, coarseness, and contrast require two or three dimensions. This suggests that the individual attributes are not infinitesimally narrow one-dimensional directions in texture space. 


\section{Varying Illuminance Condition}
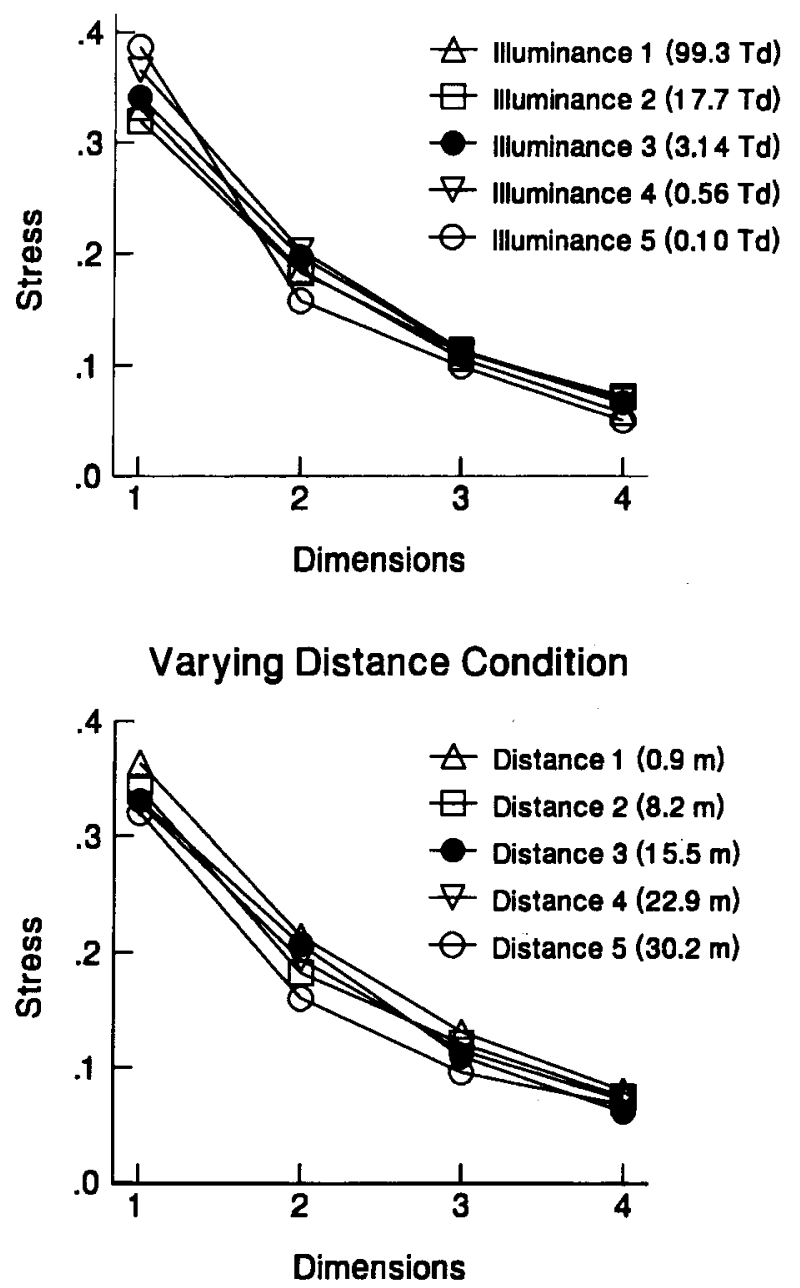

Figure 6. Supplementary experiments: overall dissimilarity judgments $(\Delta)$ from multidimensional scaling analyses of the varying illuminance and distance experiments. Stresses fell below .10 for the four-dimensional solutions. In general, the stresses were lowest for the most highly impoverished viewing conditions, suggesting a lower dimensionality in such cases.

High correlations would mean that the relative values of the judged differences are well preserved. This is the case at Distance $2\left(8.2 \mathrm{~m} ; r=.91, r^{2}=.81\right)$, with a progressive decrease in $r$ values for further distances. Figure 8 (circles) shows a similar plot for the varying illuminance condition. The correlations remain consistently high at around $r=.95$, with a sharp drop $(r=.83)$ at the lowest illuminance level $(0.1 \mathrm{Td})$. The shapes of these plots are in good agreement with the changes in the distributions of $R^{2} \mathrm{~s}$ of Figure 7-that is, for varying illuminances, a sharp reduction in performance between Illuminances 4 and 5 (Figure 7, bottom left; Figure 8, circles), and for the distance case, a generally more gradual change over the five distances (Figure 7, right; Figure 8, squares). If we take a cutoff of $r=.90$ to mark highly significant correlations, these results delimit the range over which innate judgments of overall texture dissimilarity are effectively constant: for distance, 1-8 m; for illuminance, 100-1 Td.

\section{Identification Task}

To compare the $\Delta$ and $\Delta^{A}$ dissimilarity ratings with a more objective measure of observers' perceptions, the ability to identify texture samples was studied in an ancillary experiment. From varying distances (as in the Judgments Under Impoverished Viewing Conditions section), the observers viewed panels of 20 texture samples and provided their best guesses at identifying the distant samples, referring to a hand-held panel of thumbnail samples of the textures, to avoid any confusion over names (see the Method section). All other aspects of the viewing conditions were comparable with the other experiments of the study; with 10 observers, pooled data consisted of 2,000 identifications for each of the five distances.

Data were confusion matrices: Each row contained the observers' pooled identifications for a single texture (raw data in Appendix B). When all the stimuli are equally likely, as here, it is convenient to reduce such confusion matrices to the information received in bits (Garner \& Hake, 1951). As was expected, performance was virtually perfect at $0.9 \mathrm{~m}$ (ideally 4.3 bits for 20 equally likely textures), declining smoothly to 1.6 bits at the furthest distance. For the furthest distance, both the information calculations and our regression analyses (Results 5.2) were consistent, with judgments being largely based on 3-5 shades of lightness and a dimensionality of one.

In a stimulus-response confusion matrix, accurate identifications lie on the diagonal, and errors off it. If there is a single perceptual space that is accessed by both identification and comparison tasks, identification errors for a pair of mirror-position cells (e.g., see Distance 2 in Appendix B, cells "D37 sent/D68 received" and "D68 sent/ D37 received") should be correlated with the overall dissimilarity judgment, $\Delta$, for the same texture pair in the varying distance experiment. Figure 9 shows scatter plots of error rate, pooled across all pairs of cells and all observers at the four further distances, against $\Delta$. The general inverse relation means that the higher a dissimilarity rating a pair of textures receives, the less likely it is that they will be confused in the identification task. This provides assurance that subjective ratings are related to more ecological measures of visual performance, such as identifications.

If identification errors do resemble dissimilarities to some extent, do they also imply distances in a lowdimensional perceptual space? Figure 10 shows that all solutions at each distance achieve stresses below .10 by four dimensions. The expectation that dimensionality might decrease at long distances does not, however, receive strong support. 
A Illuminance 1 (99.3 Td)

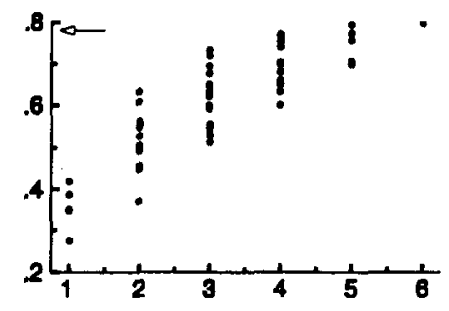

Illuminance 2 (17.7 Td)

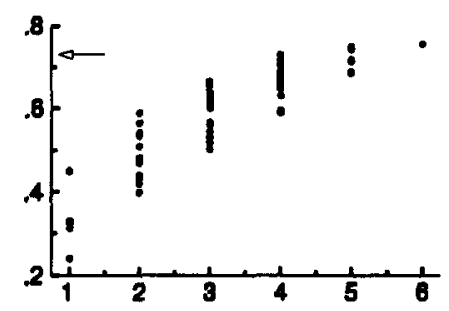

Illuminance 3 (3.14 Td)

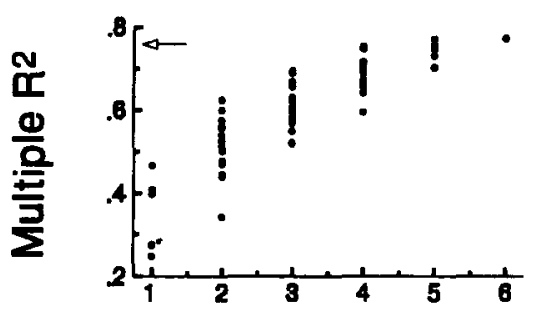

Illuminance 4 (0.56 Td)

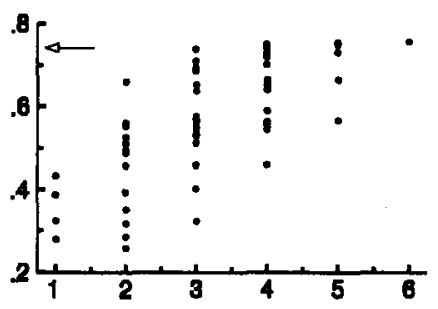

Illuminance 5 (0.10 Td)

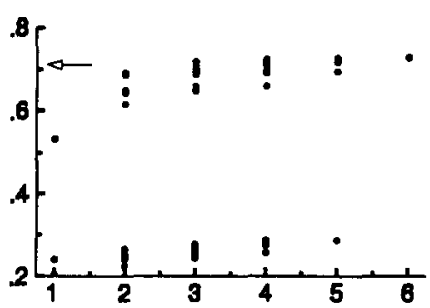

3 Distance 1 (0.9 m)

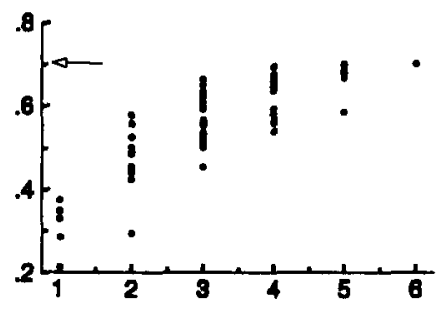

Distance $2(8.2 \mathrm{~m})$

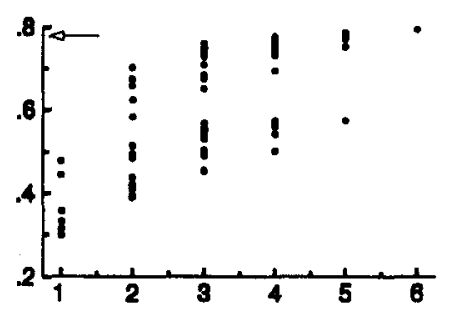

Distance $3(15.5 \mathrm{~m})$

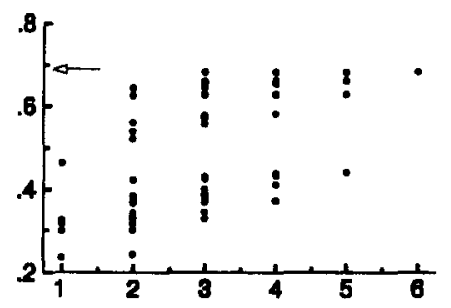

Dlstance $4(22.9 \mathrm{~m})$

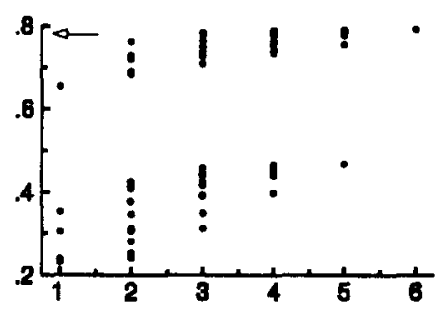

Distance 5 (30.2 m)

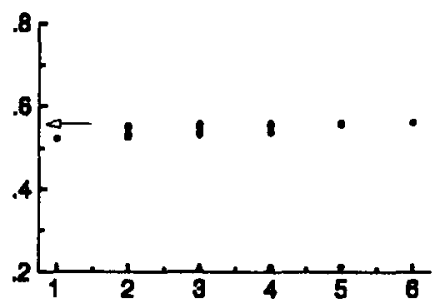

\section{Number of Attributes}

Figure 7. $\Delta$ versus $\Delta^{A}$ relationship: distance and illuminance experiments. Multiple regressions for varying viewing conditions. Regressions were performed for $\Delta$ and $\Delta^{A}$ ratings gathered for systematic decreases in illuminance (A) or increases in distance (B). All illuminance levels demonstrated high maximum $R^{2}$, which increased with added attributes and reached a plateau at around .80 (arrows denote plateaus). The $R^{2}$ s for the four highest illuminances followed a similar distribution. There was a sharp reduction in the number of useful attributes at the lowest illuminance. In that case, closer scrutiny would show that lightness accounts for most of the explained variance. There is a more gradual change in the distributions of $R^{2}$ sover distance. Again, at each distance, maximum $R^{2} s$ increased with added attributes and, in this case, reached a plateau at around .70 (arrows). At the furthest distance, there were few viable attributes, lightness explaining most of the variance. Lightness was the major variable when viewing included impoverished situations. 


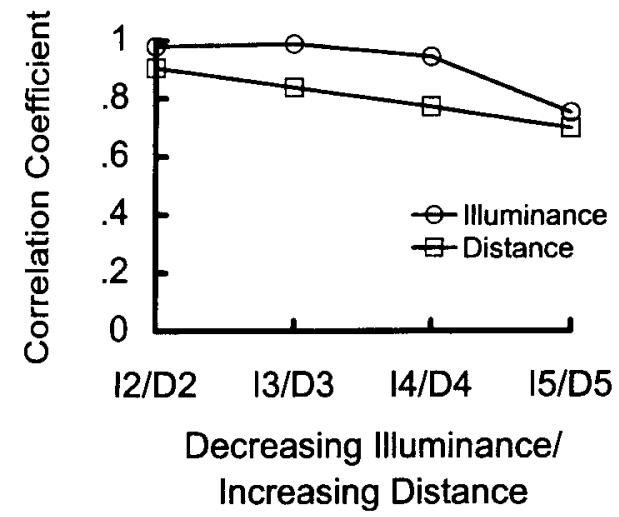

Figure 8. Overall dissimilarity judgments $(\Delta)$ : comparisons between impoverished and optimal conditions. The squares denote Pearson correlations between $\Delta$ ratings at the closest distance $(0.9 \mathrm{~m})$ and comparable ratings made at the further distances (8.2-30.2 m, or D2-D5, respectively). Distance D2 obtains high correlations, with a rapid decrease in $r$ values for further distances. The diamonds denote correlations between $\Delta$ ratings at the brightest illuminance ( $99.3 \mathrm{Td}$ ) and comparable ratings made at dimmer illuminances (17.7-0.10 Td, or L2-L5, respectively). The correlations remain consistently high at around $r=.95$, with a sharp drop at the lowest illuminance, L5.

\section{DISCUSSION}

\section{Dimensionality}

Our results show that texture space is at least fourdimensional, ${ }^{4}$ slightly higher than previous estimates, owing to our inclusion of the important attribute of texture lightness, which was omitted in previous studies. Tamura et al. (1978) and Amadasun and King (1989) made no explicit claims concerning dimensionality, but because of poor prediction of similarity judgments or correlations among attributes, respectively, their results would only permit a maximum of three dimensions. Rao and Lohse $(1993,1996)$ asked observers to perform rating and grouping tasks on a Brodatz texture set and concluded that judgments could be based on as few as three dimensions.

Despite the possibility that other viable attributes may yet be discovered, the true dimensionality may not be much more than four. Coarse rapid judgments (i.e., our ratings and the grouping tasks of Rao \& Lohse, 1996) do not reduce the observed dimensionality, because our identification task yields a similar estimate. However, even with greatly refined judgments, it is rather unlikely that coordinate values for, say, coarseness, regularity, contrast, and lightness would ever recover the appearance of a texture in the manner that hue, saturation, and brightness recover color. A much larger texture set might stress dimensions that we have missed, but this trend is not observed when comparing the results of our study with those of Rao and Lohse (1993, 1996), despite differing set sizes.

Judgments on any texture set, then, whether gratings or textured materials, may yield low-dimensional representations (Table 4). Although texture sensing (Field, 1987;
Sakitt \& Barlow, 1982) may actually possess high dimensionality, a much lower dimensional attention-limited "working space" may suffice for practical judgments in local environments (Attneave, 1974; Broadbent, 1958; Harvey \& Gervais, 1978; Tsotsos, 1990). The selection of relevant attributes would then be highly contingent on the structure in the environment. For example, discriminations of a set of wood-grains (Brodatz, 1972) would almost certainly yield a new subspace. Task must also be important. Even with a particular set of textures, observers differentially weight dimensions in order to optimize their performance on different tasks (see, e.g., Getty et al., 1979).

\section{Attributes}

We have found six reliable candidate attributes for directions in texture space, especially coarseness and regularity, and also lightness when viewing under impoverished conditions. Because of correlations between some of the attributes, 4-D models suffice. We obtained overall dissimilarity ratings before making attribute ratings, to avoid bias. The reversed order of the tasks in previous studies made the validity of their attributes less certain (Amadasun \& King, 1989; Rao \& Lohse, 1996; Tamura et al., 1978).

Coarseness has long been recognized as a fundamental textural feature, and its primacy in our observers' judgments is not surprising (Table 4; Hallett \& Hofmann, 1991). The importance of regularity may be a measure of the visual system's weightings of two functionally distinct approaches to texture analysis - namely, statistical versus structural (Haralick, 1979; Tyler \& Chang, 1977). Directionality's importance is comparable to that of regularity and was one of the strongest cues for similarity judgments in the study by Tamura et al. (1978). Texture lightness was examined because it has a clear physical correlate in spatial mean of surface reflectance and seems likely to be necessary in everyday comparisons of monochrome texture pairs. In the event, lightness proved to be a useful predictor of dissimilarity, particularly for widely ranging viewing conditions. None of the previous perceptual studies has explored lightness - even as a confounding factor. Contrast and edginess, although useful, were the two least significant attributes. Their significant correlation was already noted by Tamura et al. Measures of contrast, by themselves, were never the most important predictors of similarity in previous perceptual studies.

Four of the attributes - coarseness, regularity, lightness, and contrast - attained near-orthogonality (Table 3) and, since they were all useful predictors of overall dissimilarity, might serve as candidate cardinal axes for a 4-D texture space (directionality and edginess would assume nonorthogonal orientations). But there were no a priori grounds for assuming that cardinal axes would lend themselves to labeling; the English language may lack the proper adjectives. This was evidenced by Rao and Lohse's (1996) difficulty in producing one-adjective labels for all their axes-for example, "granular/coarse/low- 


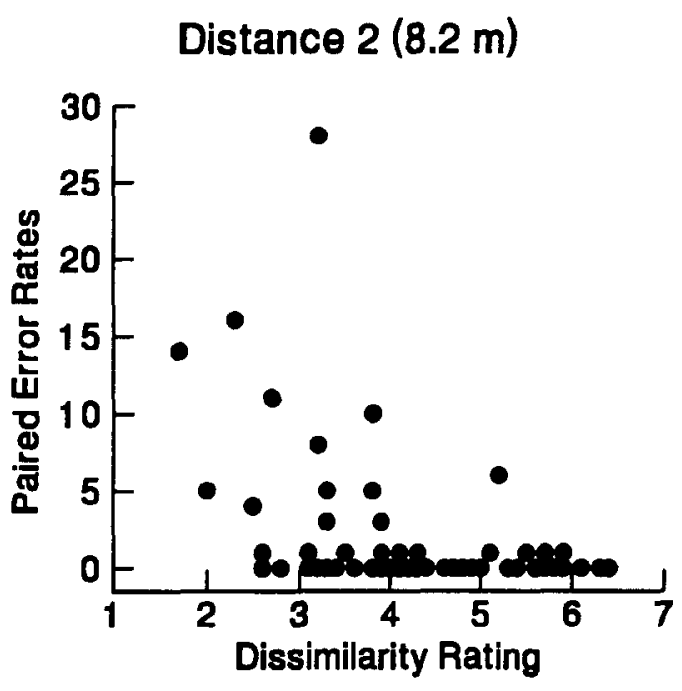

Distance $4(22.9 \mathrm{~m})$

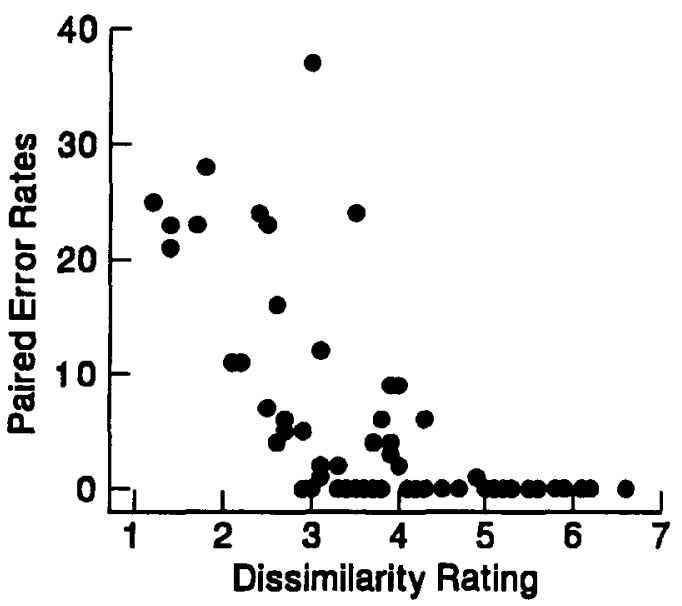

Distance $3(15.5 \mathrm{~m})$

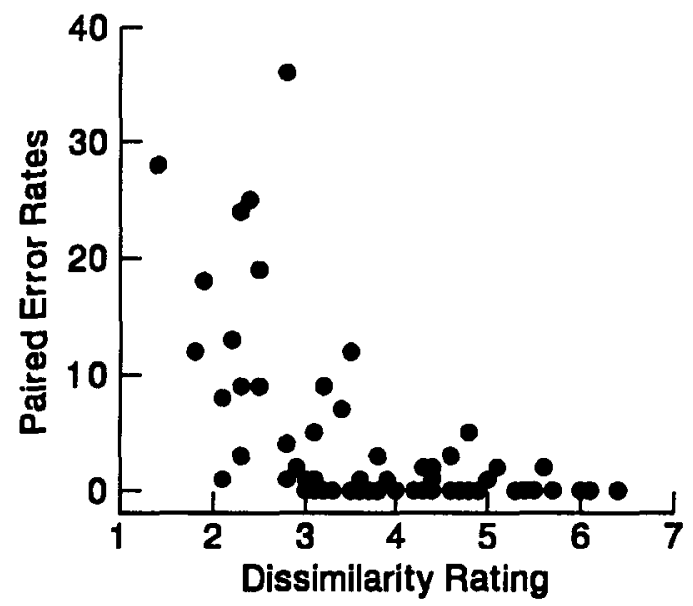

Distance $5(30.2 \mathrm{~m})$

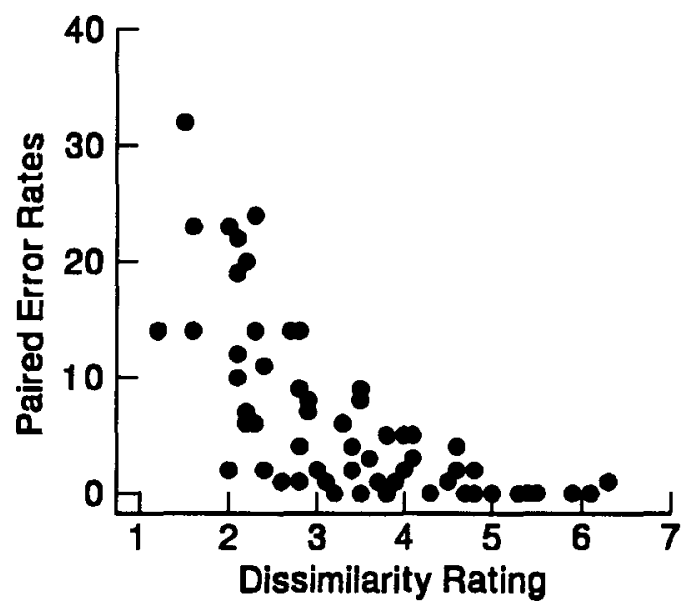

Figure 9. Identification-dissimilarity relationship for the four longer distances. The ordinate shows errors of confusion in the identification task for the 12 textures that were studied in both the identification and the overall dissimilarity $(\Delta)$ tasks. The abscissa is the mean overall dissimilarity $(\Delta)$ ratings given in the Judgments Under Impoverished Viewing Conditions section.

complexity." Related to the issue of cardinality is our suggestion that a portion of the unexplained variance may be due to the attributes' not being truly unidimensional. Multidimensionality is implied in computational definitions (Amadasun \& King, 1989; Tamura et al., 1978). For example, the definition of coarseness is 2-D, since it involves both the size and the spacing of repeating elements.

Perhaps, our view of how attributes contribute to dissimilarity judgments is too simplistic. On the whole, we think not. Admittedly, both Tamura et al. (1978) and Amadasun and King (1989) experienced only moderate success in predicting texture similarities by calculating two measures of similarity-namely, the minimum Mahalanobis and Euclidian distances. But Rao and Lohse
(1996) achieved reasonable predictions with their classification and regression tree (Brieman, Friedman, Olshen, \& Stone, 1984) and discriminant analyses, with estimated "true" classification rates of $70 \%$ and $49 \%$, respectively. Tibshirani kindly examined our varying-distance experiment from the viewpoint of general additive models (Hastie \& Tibshirani, 1990), with slightly improved fits in only a few instances. Other possible approaches to modeling include (1) an exhaustive search for the optimal metric in our MDS analyses or (2) trajectory mapping, a technique that traces paths through a feature space that is not necessarily metric or homogeneous (Richards \& Koenderink, 1993). But since we can make valid predictions with simple models, it would seem that gains from any of the above elaborations will be small. 


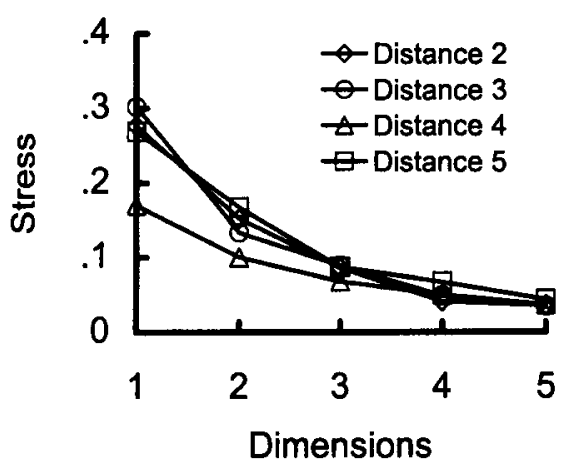

Figure 10. Stresses for multidimensional scaling configurations derived from identifications. All the distances achieve stresses below .10 by three to four dimensions. Distance 1 could not be analyzed, because there were almost no errors in identification.

\section{Variability of Texture Space}

Variability across texture subsets. It is difficult to speculate how much of the unaccounted-for variance is due to inhomogeneity of the texture space and not to inadequacy of the model, intersubject variability, and so forth. But the low average prediction error from the across-set cross-validations, of the order of 1 rating unit, indicates that there is relative homogeneity across texture space.

Intersubject variability. To date, no studies of natural textures (Table 4) have explicitly examined intersubject variability. In color-naming tasks, color-normal observers are consistent individually and achieve consensus as a group, even displaying cross-cultural agreement (Boynton \& Olson, 1987; Uchikawa \& Boynton, 1987). We were, nevertheless, surprised at the relatively good agreement across observers; our ratings were less variable than was expected.

Task dependence. Does the perceptual space for textures depend on task? The strength of the associations between the two tasks of our main experiment, the overall dissimilarity and attribute dissimilarity judgments, suggests that we can posit a single space that yields different types of texture judgments. The identification task lends further evidence, because dissimilar textures were rarely confused. The dimensionalities of the MDS configurations based on transformed stimulus-response matrices are also generally low (3-4).

Table 4

Studies of Comparative Judgments of Textures

\begin{tabular}{|c|c|c|c|}
\hline $\begin{array}{c}\text { Study, Task, and Sample Size } \\
\text { (Subjects/Textures) }\end{array}$ & $\begin{array}{c}\text { Predictor } \\
\text { Characteristics }\end{array}$ & Analysis & Dimensionality \\
\hline \multicolumn{4}{|c|}{ Artificial Textures } \\
\hline $\begin{array}{l}\text { Harvey and Gervais (1978) } \\
\text { Groupings }(20 / 30)\end{array}$ & Spatial filter activities & $\begin{array}{l}\text { Discriminant analysis } \\
\text { Multidimensional scaling (MDS) } \\
\text { Canonical correlation }\end{array}$ & $\begin{array}{l}4 \text { channels } \\
3 \text { MDS axes }\end{array}$ \\
\hline $\begin{array}{l}\text { Harvey and Gervais ( } 1981) \\
\text { Triadic }(2 / 30) \text { and paired comparisons }(6 / 30\end{array}$ & Spatial filter activities and power spectra & $\begin{array}{l}\text { MDS } \\
\text { Regressions } \\
\text { Canonical correlation }\end{array}$ & $\begin{array}{l}4 \text { channels } \\
3 \text { MDS }\end{array}$ \\
\hline $\begin{array}{l}\text { Kahana and Bennett (1994) } \\
\text { Classification }(16 / 20)\end{array}$ & Differences in filter phases & $\begin{array}{l}\text { MDS } \\
\text { Regressions }\end{array}$ & 2 channels \\
\hline \multicolumn{4}{|c|}{ Natural Textures } \\
\hline $\begin{array}{l}\text { Tamura, Mori, and Yamawaki (1978) } \\
\text { Paired comparisons }(48 / 16)\end{array}$ & $\begin{array}{l}\text { Coarseness } \\
\text { Contrast } \\
\text { Directionality } \\
\text { Line-likeness } \\
\text { Regularity } \\
\text { Roughness }\end{array}$ & $\begin{array}{l}\text { Thurstonian scaling } \\
\text { Correlations } \\
\text { Nearest texture prediction }\end{array}$ & $\leq 6($ not specified $)$ \\
\hline $\begin{array}{l}\text { Amadasun and King ( } 1989) \\
\text { Rankings and texture similarities }(88 / 10)\end{array}$ & $\begin{array}{l}\text { Coarseness } \\
\text { Contrast } \\
\text { Busyness } \\
\text { Complexity } \\
\text { Texture strength }\end{array}$ & $\begin{array}{l}\text { Rank correlations } \\
\text { Nearest texture prediction }\end{array}$ & $\leq 5$ (not specified) \\
\hline $\begin{array}{l}\text { Rao and Lohse (1993) } \\
\text { Groupings }(20 / 30)\end{array}$ & $\begin{array}{l}\text { Periodicity } \\
\text { Directionality } \\
\text { Complexity }\end{array}$ & $\begin{array}{l}\text { MDS } \\
\text { Hierarchical cluster analysis }\end{array}$ & 3 MDS axes \\
\hline $\begin{array}{l}\text { Rao and Lohse (1996) } \\
\text { Groupings and ratings (20/56) }\end{array}$ & $\begin{array}{l}\text { Repetitive } \\
\text { High-contrast/nondirectional } \\
\text { Granular/coarse/low-complexity }\end{array}$ & $\begin{array}{l}\text { MDS } \\
\text { Regression trees } \\
\text { Discriminant analysis } \\
\text { Principal component analysis }\end{array}$ & 3 MDS axes \\
\hline $\begin{array}{l}\text { Present study } \\
\quad \text { Paired comparisons }(12 /[5 \text { sets of } 12])\end{array}$ & $\begin{array}{l}\text { Coarseness } \\
\text { Regularity } \\
\text { Contrast } \\
\text { Lightness } \\
\text { Directionality } \\
\text { Edginess }\end{array}$ & $\begin{array}{l}\text { MDS } \\
\text { Regressions } \\
\text { Cross-validation } \\
\text { Redundancy analysis }\end{array}$ & 4 MDS axes \\
\hline
\end{tabular}




\section{Summary and Conclusions}

We have attempted to characterize regularities in observers' comparisons of complex, natural textures, invoking the notion of a texture space and asking questions concerning the dimensionality and axes of such a space. It seems that at least four dimensions are required to explain the judgments. Comparisons of this dimensionality with that of other studies suggests that increases in texture set size do not necessarily increase dimensionality; the dimensionality may not be much greater than four. All of the studied attributes proved useful, with coarseness, regularity, contrast, and lightness possibly serving as candidate orthogonal axes for a 4-D representation. Coarseness, regularity, and contrast have found prior use in the literature. The spatial attribute of texture lightness has not been examined before, and its relevance to texture comparisons may explain why our dimensionality estimate is slightly higher than that of previous work. There is also a suggestion that some of the attributes themselves are not strictly unidimensional. We departed from previous studies in our task ordering: Our observers were asked to make the judgments of overall dissimilarity before the attribute judgments, so that the latter would not bias the former. Intersubject variability is another issue that has not received explicit treatment to date. Our findings show that, despite using a seemingly subjective rating task, there is good agreement between observers' texture comparisons. Finally, we examined judgments of textures under a wide range of viewing conditions and with an identification task. The results suggest that there is a perceptual representation that is common to different tasks and is insensitive to reasonably wide changes in illumination and viewing distance ( $1-8 \mathrm{~m}$ and $1-90$ or more $\mathrm{Td}$ ).

\section{REFERENCES}

Amadasun, M., \& King, R. (1989). Textural features corresponding to textural properties. IEEE Transactions on Systems, Man, \& Cybernetics, 19, 1264-1274.

Attneave, F. (1974). How do we know? American Psychologist, 29, 493-499.

BENDZSACK, A. (1996). The effect of varying luminance levels on the perception of monochrome textures. Unpublished undergraduate thesis, University of Toronto, Toronto, Ontario, Canada.

Bhushan, N., RaO, A. R., \& Lohse, G. L. (1997). The texture lexicon: Understanding the categorization of visual texture terms and their relationship to texture images. Cognitive Science, 21, 219-246.

Boynton, M., \& Olson, C. X. (1987). Locating basic colors in the OSA space. Color Research \& Applications, 12, 94-105.

Brieman, L., Friedman, J., Olshen, R., \& Stone, C. (1984). Classification and regression trees. Pacific Grove, CA: Wadsworth \& Brooks.

Broadbent, D. E. (1958). Perception and communication. New York: Pergamon.

Brodatz, P. (1966). Textures. New York: Dover.

Brodatz, P. (1972). Wood and wood grains. New York: Dover.

CAMPBELL, F. W., \& RoBson, J. G. (1968). Application of Fourier analysis to the visibility of gratings. Journal of Physiology, 203, 55 1-566.

Cront.y-Dillon, J. (Series Ed.), \& Gouras, P. (Vol. Ed.) (1991). Vision and visual dysfunction: Vol. 6. The perception of colour. Hampshire, U.K.: MacMillan.

DAUCimaN. J. (1987). Image analysis and compact encoding by 2D
Gabor primitives. Proceedings of the SPIE-The International Society for Optical Engineering, 758, 19-30.

De Valois, R. L., Albrecht, D. G., \& Thorell, L. G. (1982). Spatial frequency selectivity of cells in macaque visual cortex. Vision Research, 22, 545-559.

Efron, B., \& Tibshirani, R. J. (1993). An introduction to the bootstrap. Toronto: Chapman \& Hall.

FIELD, D. J. (1987). Relations between the statistics of natural images and the response properties of cortical cells. Journal of the Optical Society of America A, 4, 2379-2394.

FLEET, D., \& JEPSON, A. D. (1989). On the hierarchical construction of orientation and velocity sensitive filters. IEEE Transactions in Pattern Analysis \& Machine Intelligence, 2, 315-325.

GaRnER, W. R., \& HAKE, H. W. (1951). The amount of information in absolute judgments. Psychological Review, 58, 446-459.

GetTy, D. J., Swers, J. A., Swets, J. B., \& David, M. G. (1979). On the prediction of confusion matrices from similarity judgments. Perception \& Psychophysics, 26, 1-19.

Hallett, P. E., \& Hofmann, M. I. (1991). Segregation of some meshderived textures evaluated by free viewing. Vision Research, 31, 1701-1716.

HARALICK, R. M. (1979). Statistical and structural approaches to texture. Proceedings of the IEEE, 67, 786-804.

HaRVEY, L. O., JR., \& Gervais, M. J. (1978). Visual texture perception and Fourier analysis. Perception \& Psychophysics, 24, 534-542.

HARVEY, L. O., JR., \& Gervais, M. J. ( 1981 ). Internal representation of visual texture as the basis for the judgment of similarity. Journal of Experimental Psychology, 7, 741-753.

Hastie, T. J., \& TibshiRani, R. J. (1990). Generalized additive models. New York: Chapman \& Hall.

Hofmann, M. J., \& Hallett, P. E. (1993a). Preattentive discrimination of relative phase modeled by interacting Gabor or difference-ofGaussian filters. Vision Research, 33, 2569-2587.

Hofmann, M. I., \& HalletT, P. E. (1993b). Texture segregation based on two-dimensional relative phase differences in composite sinewave grating patterns. Vision Research, 33, 221-234.

JuLESz, B. (1981). Textons, the elements of texture perception, and their interactions. Nature, 290, 91-97.

Kahana, M. J., \& BenNetT, P. J. (1994). Classification and perceived similarity of compound gratings that differ in relative spatial phase. Perception \& Psychophysics, 55, 642-656.

KRUSKALL, J. B. (1964). Multidimensional scaling by optimizing goodness of fit to a nonmetric hypothesis. Psychometrika, 29, 1-27.

Kruskall, J. B., \& Wish, M. (1978). Multidimensional scaling. Beverley Hills, CA: Sage.

Malik, J., \& Perona, P. (1990). Preattentive texture discrimination with early vision mechanisms. Journal of the Optical Society of America, 7, 923-932.

Nikmanesh, H. (1996). Contribution of textural attributes to texture perception at varying distances. Unpublished undergraduate thesis, University of Toronto, Toronto, Ontario, Canada.

RAO, A. R., \& LOHSE, G. L. (1993). Identifying high level features of texture perception. CGVIP: Graphical Models \& Image Processing, 55, 218-233.

RAO, A. R., \& LoHSE, G. L. (1996). Towards a texture naming system: Identifying relevant dimensions of texture. Vision Research, 36 , 1649-1669.

RICHARDS, W. (1979). Quantifying sensory channels: Generalizing colorimetry to orientation and texture, touch and tones. Sensory Processes, 3, 207-229.

Richards, W, \& Koenderink, J. J. (1993). Trajectory mapping: A new nonmetric scaling technique (Occasional Paper 48). Cambridge, MA: Massachusetts Institute of Technology, Center for Cognitive Science.

RichaRDS, W., \& POLIT, A. (1974). Texture matching. Kybernetik, 16, 155-162.

Ross, R. T. (1934). Optimum orders for the presentation of pairs in the method of paired comparisons. Journal of Educational Psychology, 25, 375-382. 
SakitT, B., \& Barlow, H. B. (1982). A model for the economical encoding of the visual image in cerebral cortex. Biological Cybernetics, 43, 97-108.

SChWARTZ, G. (1978). Estimating the dimension of a model. Annals of Statistics, 6, 461-464.

Shiffman, S. S., Reynolds, M. L., \& Young, F. W. (1981). Handbook of multidimensional scaling. New York: Academic Press.

STEWART, D., \& Love, W. (1968). A general canonical correlation index. Psychological Bulletin, 70, 160-163.

Tamura, H., Mori, S., \& Yamawaki, T. (1978). Textural features corresponding to visual perception. IEEE Transactions on Systems, Man, \& Cybernetics, SMC-8, 460-473.

Tsotsos, J. K. (1990). Analyzing vision at the complexity level. Behavioral \& Brain Sciences, 13, 423-469.

Tyler, C. W., \& Chang, J. J. (1977). Visual echoes: The perception of repetition in quasi-random patterns. Vision Research, 17, 109-116.

UChIKaWA, K., \& Boynton, R. M. (1987). Categorical color perception of Japanese observers: Comparison with that of Americans. Vision Research, 27, 1825-1833.

\section{NOTES}

1. In response to a reviewer's comment that lightness could be seen as a mere scale factor, it must be noted that texture lightness has little or noth- ing to do with a noisy environmental variable such as lighting or illumination. We point out that a material with a lower mean reflectance, whether monochrome or spectral reflectance, is a physically or chemically different material or, at least, a dirty or camouflaged one. In any case, the material would be assessed as being texturally different. For instance, a human subject might wonder whether the material would feel different when touched. An automated texture analysis program used, say, for recognition of pathologic tissue in medical imaging or of defective materials in industrial quality control would also likely be expected to make discriminations on the basis of lightness assessments or mean reflectance.

2. For brevity, the attributes are sometimes referred to by their first letters $(\mathrm{l}, \mathrm{c}, \mathrm{e}, \mathrm{f}, \mathrm{d}, \mathrm{r})$. To distinguish it from contrast, coarseness will be denoted by "f " for "fineness."

3. By texture contrast, we refer to the more complex assessment of the overall contrast within a texture, rather than to a simple grating contrast or $S D /$ mean for pixel gray-level values.

4. We agree with a reviewer's comment that the number of subjects involved in our main experiment $(n=12)$ may be too few to make strong claims about dimensionality in the population at large. However, we examined around 40 subjects when pooling across all of our experiments and did not notice any striking intersubject variability over and above that presented in this paper. Furthermore, involvement of more subjects in the main experiment would, if anything, be likely to increase our estimate of dimensionality (four, this being our best estimate of its lower limit).

\section{APPENDIX A \\ Brodatz (1966) Textures Used as Stimuli}

\begin{tabular}{llllll} 
& \multicolumn{1}{c}{ Set 1 } & \multicolumn{1}{c}{ Set 2 } & \multicolumn{1}{c}{ Set 3 } \\
\hline D9 & grass lawn & D2 & fieldstone & D4 & pressed cork \\
D20 & French canvas & D5 & expanded mica & D12 & bark of tree \\
D22 & reptile skin & D11 & woolen cloth & D14 & woven wire \\
D29 & beach sand & D35 & lizard skin & D17 & herringbone weave \\
D34 & netting & D49 & straw screening & D18 & raffia weave \\
D52 & oriental straw cloth & D55 & straw matting & D21 & French canvas \\
D66 & plastic pellets & D64 & oriental rattan & D31 & beach pebbles \\
D68 & wood grain & D70 & wood grain & D50 & raffia \\
D87 & sea fan & D73 & soap bubbles & D60 & European marble \\
D93 & fur & D92 & pigskin & D82 & oriental straw cloth \\
D98 & crushed rose quartz & D101 & cane & D107 & Japanese rice paper \\
D112 & plastic bubbles & D109 & handmade paper & D111 & plastic bubbles \\
& \multicolumn{1}{c}{ Set 4 } & & \multicolumn{1}{c}{ Set 5 } & & \\
\hline D1 & woven wire & D6 & woven wire & & \\
D23 & beach pebbles & D7 & fieldstone & & \\
D24 & calf leather & D15 & straw & & \\
D32 & pressed cork & D19 & woolen cloth & & \\
D62 & European marble & D27 & beach sand and pebbles & \\
D65 & oriental rattan & D37 & water & & \\
D72 & tree stump & D47 & woven brass mesh & \\
D77 & cotton canvas & D74 & coffee beans & \\
D80 & oriental straw cloth & D79 & oriental grass fibre cloth & \\
D83 & woven matting & D84 & raffia & & \\
D100 & ice crystals & D86 & ceiling tile & & \\
D104 & loose burlap & D105 & cheesecloth & & \\
\hline
\end{tabular}

Note-The letter/number combinations follow the nomenclature used by Brodatz (1966). 
APPENDIX B

Stimulus-Response Matrices for the Observers' Pooled Responses for Each Distance (1-5)

\section{RESPONSE (Distance $1,0.9 \mathrm{~m}$ )}

\begin{tabular}{|c|c|c|c|c|c|c|c|c|c|c|c|c|c|c|c|c|c|c|c|c|}
\hline \multirow[b]{2}{*}{2} & 4 & 9 & 12 & 16 & 20 & 22 & 29 & 34 & 37 & 52 & 55 & 66 & 68 & 76 & 86 & 87 & 93 & 98 & 109 & 112 \\
\hline & 99 & 1 & 0 & 0 & 0 & 0 & 0 & 0 & 0 & 0 & 0 & 0 & 0 & 0 & 0 & 0 & 0 & 0 & 0 & 0 \\
\hline 9 & 1 & 97 & 0 & 0 & 1 & 0 & 0 & 0 & 0 & 0 & 0 & 0 & 0 & 0 & 0 & 0 & 1 & 0 & 0 & 0 \\
\hline 12 & 0 & 0 & 100 & 0 & 0 & 0 & 0 & 0 & 0 & 0 & 0 & 0 & 0 & 0 & 0 & 0 & 0 & 0 & 0 & 0 \\
\hline 16 & 0 & 0 & 0 & 99 & 0 & 0 & 0 & 0 & 0 & 0 & 0 & 0 & 1 & 0 & 0 & 0 & 0 & 0 & 0 & 0 \\
\hline 20 & 0 & 0 & 0 & 0 & 100 & 0 & 0 & 0 & 0 & 0 & 0 & 0 & 0 & 0 & 0 & 0 & 0 & 0 & 0 & 0 \\
\hline 22 & 0 & 0 & 0 & 0 & 10 & 0 & 0 & 0 & 0 & 0 & 0 & 0 & 0 & 0 & 0 & 0 & 0 & 0 & 0 & 0 \\
\hline 29 & 3 & 1 & 0 & 0 & 0 & 0 & 96 & 0 & 0 & 0 & 0 & 0 & 0 & 0 & 0 & 0 & 0 & 0 & 0 & 0 \\
\hline 34 & 0 & 0 & 0 & 0 & 0 & 0 & 0 & 99 & 0 & 1 & 0 & 0 & 0 & 0 & 0 & 0 & 0 & 0 & 0 & 0 \\
\hline 37 & 0 & 0 & 0 & 0 & 0 & 0 & 0 & 0 & 100 & 0 & 0 & 0 & 0 & 0 & 0 & 0 & 0 & 0 & 0 & 0 \\
\hline 52 & 0 & 0 & 0 & 0 & 0 & 0 & 0 & 0 & 0 & 100 & 0 & 0 & 0 & 0 & 0 & 0 & 0 & 0 & 0 & 0 \\
\hline 55 & 0 & 0 & 0 & 2 & 0 & 0 & 0 & 0 & 0 & 0 & 98 & 0 & 0 & 0 & 0 & 0 & 0 & 0 & 0 & 0 \\
\hline 66 & 0 & 0 & 0 & 0 & 0 & 0 & 0 & 0 & 0 & 0 & 0 & 100 & 0 & 0 & 0 & 0 & 0 & 0 & 0 & 0 \\
\hline 68 & 0 & 0 & 0 & 0 & 0 & 0 & 0 & 0 & 0 & 0 & 0 & 0 & 100 & 0 & 0 & 0 & 0 & 0 & 0 & 0 \\
\hline 76 & 0 & 0 & 0 & 1 & 0 & 0 & 0 & 0 & 0 & 0 & 0 & 0 & 1 & 98 & 0 & 0 & 0 & 0 & 0 & 0 \\
\hline 86 & 1 & 0 & 0 & 0 & 0 & 0 & 0 & 0 & 0 & 0 & 1 & 0 & 0 & 0 & 97 & 0 & 0 & 0 & 1 & 0 \\
\hline 87 & 0 & 0 & 0 & 0 & 0 & 0 & 0 & 0 & 0 & 0 & 0 & 0 & 0 & 0 & 0 & 100 & 0 & 0 & 0 & 0 \\
\hline 93 & 0 & 0 & 0 & 0 & 0 & 0 & 0 & 0 & 0 & 0 & 0 & 0 & 0 & 0 & 0 & 0 & 100 & 0 & 0 & 0 \\
\hline 98 & 0 & 0 & 0 & 0 & 0 & 0 & 0 & 0 & 0 & 0 & 0 & 0 & 0 & 0 & 0 & 0 & 0 & 100 & 0 & 0 \\
\hline 109 & 0 & 0 & 0 & 0 & 0 & 0 & 0 & 0 & 0 & 0 & 0 & 0 & 0 & 0 & 0 & 0 & 0 & 0 & 100 & 0 \\
\hline 112 & 0 & 0 & 0 & 0 & 0 & 0 & 0 & 0 & 0 & 0 & 0 & 0 & 0 & 0 & 0 & 0 & 0 & 0 & 0 & 100 \\
\hline
\end{tabular}

RESPONSE (Distance 2, $8.2 \mathrm{~m}$ )

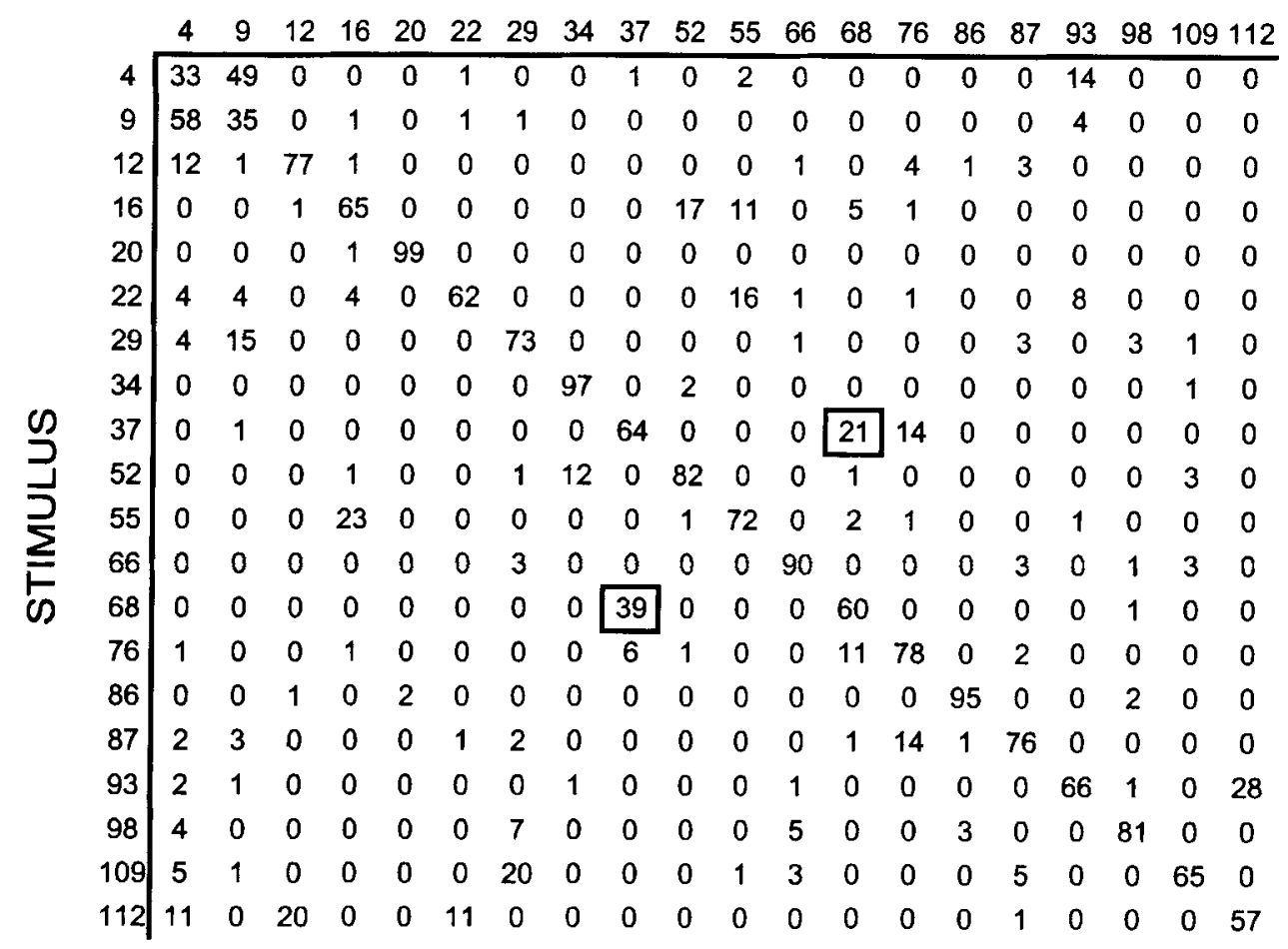


RESPONSE (Distance 3, $15.5 \mathrm{~m}$ )

\begin{tabular}{|c|c|c|c|c|c|c|c|c|c|c|c|c|c|c|c|c|c|c|c|c|}
\hline & 4 & 9 & 12 & 16 & 20 & 22 & 29 & 34 & 37 & 52 & 55 & 66 & 68 & 76 & 86 & 87 & 93 & 98 & 109 & 112 \\
\hline 4 & 8 & 21 & 0 & 31 & 1 & 3 & 0 & 0 & 0 & 1 & 22 & 0 & $\overline{1}$ & 0 & 0 & 0 & 12 & 0 & 0 & 0 \\
\hline 9 & 51 & 20 & 2 & 3 & 0 & 5 & 2 & 0 & 0 & 0 & 5 & 0 & 0 & 0 & 1 & 1 & 9 & 1 & 0 & 0 \\
\hline 12 & 13 & 1 & 64 & 0 & 0 & 0 & 0 & 0 & 0 & 0 & 0 & 0 & 1 & 3 & 3 & 12 & 0 & 3 & 0 & 0 \\
\hline 16 & 0 & 13 & 0 & 43 & 3 & 2 & 8 & 0 & 3 & 14 & 2 & 0 & 11 & 1 & 0 & 0 & 0 & 0 & 0 & 0 \\
\hline 20 & 0 & 2 & 0 & 1 & 81 & 0 & 0 & 0 & 1 & 7 & 6 & 0 & 1 & 0 & 0 & 0 & 0 & 0 & 0 & 1 \\
\hline 22 & 19 & 13 & 0 & 3 & 0 & 19 & 0 & 0 & 0 & 0 & 19 & 0 & 0 & 2 & 0 & 1 & 19 & 0 & 0 & 5 \\
\hline 29 & 1 & 7 & 0 & 0 & 1 & 0 & 70 & 0 & 1 & 4 & 1 & 5 & 5 & 0 & 0 & 1 & 0 & 3 & 1 & 0 \\
\hline 34 & 0 & 0 & 0 & 0 & 0 & 0 & 0 & 95 & 0 & 0 & 0 & 0 & 0 & 0 & 1 & 0 & 0 & 0 & 4 & 0 \\
\hline 37 & 0 & 2 & 0 & 0 & 0 & 0 & 0 & 0 & 17 & 0 & 0 & 2 & 20 & 52 & 4 & 2 & 0 & 0 & 0 & 1 \\
\hline 52 & 0 & 0 & 0 & 0 & 0 & 0 & 9 & 28 & 2 & 45 & 0 & 1 & 3 & 0 & 1 & 0 & 0 & 0 & 11 & 0 \\
\hline 55 & 1 & 0 & 0 & 13 & 0 & 9 & 0 & 0 & 0 & 2 & 67 & 1 & 1 & 1 & 0 & 0 & 5 & 0 & 0 & 0 \\
\hline 66 & 0 & 0 & 0 & 0 & 0 & 0 & 7 & 1 & 1 & 7 & 0 & 42 & 1 & 0 & 2 & 5 & 0 & 0 & 34 & 0 \\
\hline 68 & 1 & 1 & 0 & 1 & 1 & 0 & 0 & 0 & 53 & 0 & 1 & 0 & 23 & 13 & 2 & 1 & 0 & 3 & 0 & 0 \\
\hline 76 & 3 & 2 & 0 & 1 & 7 & 4 & 1 & 0 & 5 & 1 & 0 & 0 & 18 & 44 & 1 & 7 & 0 & 6 & 0 & 0 \\
\hline 86 & 0 & 0 & 0 & 0 & 1 & 0 & 0 & 1 & 0 & 0 & 0 & 4 & 0 & 0 & 89 & 4 & 0 & 1 & 0 & 0 \\
\hline 87 & 3 & 3 & 0 & 0 & 0 & 0 & 2 & 0 & 19 & 1 & 1 & 4 & 18 & 26 & 3 & 14 & 2 & 4 & 0 & 0 \\
\hline 93 & 2 & 0 & 3 & 0 & 0 & 6 & 0 & 0 & 0 & 0 & 2 & 0 & 0 & 0 & 0 & 0 & 50 & 2 & 0 & 35 \\
\hline 98 & 10 & 1 & 12 & 0 & 0 & 0 & 2 & 0 & 0 & 0 & 1 & 1 & 0 & 3 & 5 & 8 & 0 & 57 & 0 & 0 \\
\hline 109 & 0 & 0 & 0 & 0 & 0 & 0 & 6 & 0 & 0 & 0 & 0 & 25 & 0 & 1 & 0 & 7 & 0 & 0 & 61 & 0 \\
\hline 112 & 1 & 0 & 17 & 0 & 1 & 19 & 0 & 0 & 0 & 0 & 0 & 0 & 0 & 1 & 0 & 1 & 1 & 0 & 0 & 59 \\
\hline
\end{tabular}

RESPONSE (Distance 4, $22.9 \mathrm{~m}$ )

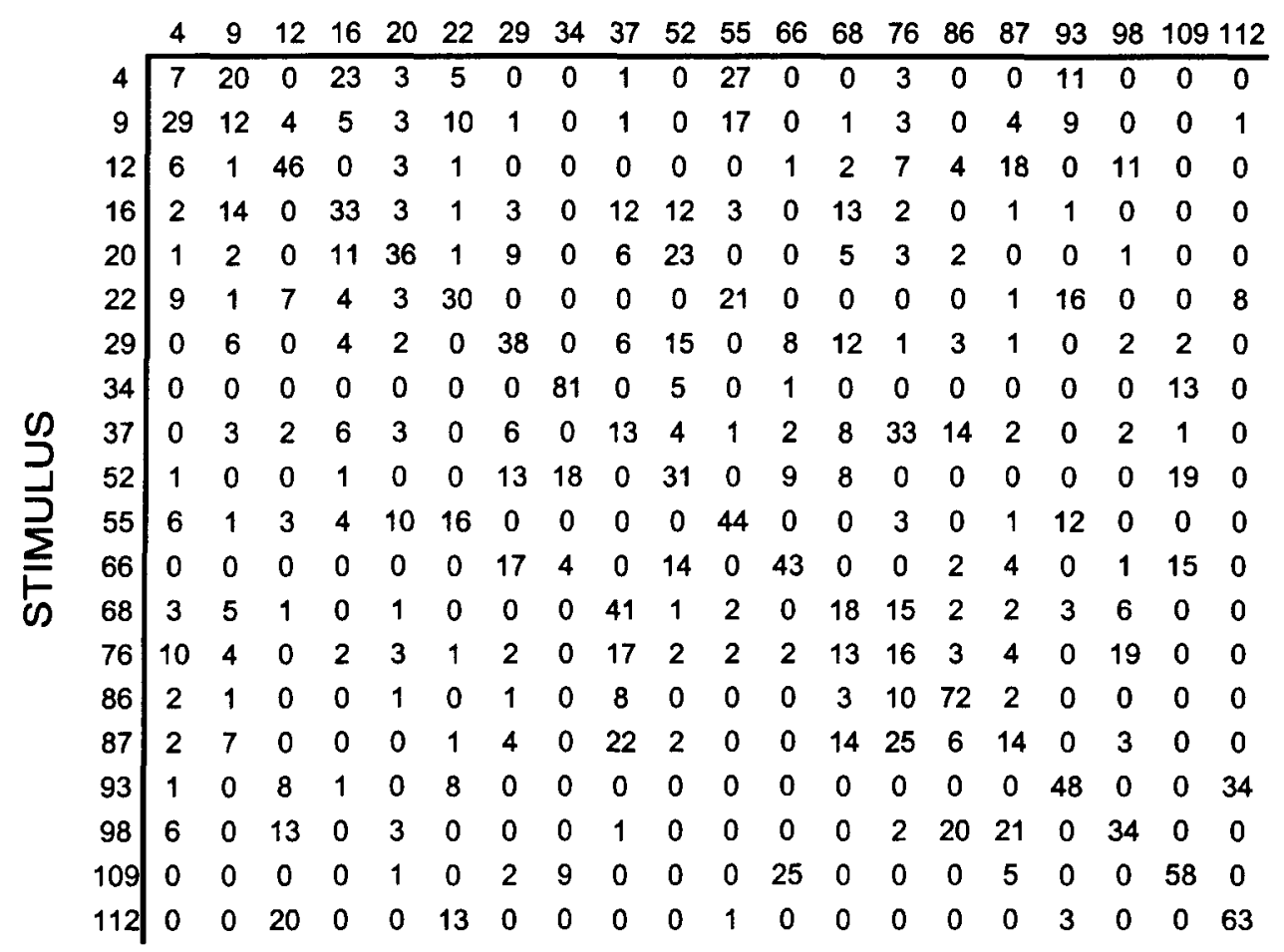


RESPONSE (Distance 5, $30.2 \mathrm{~m}$ )

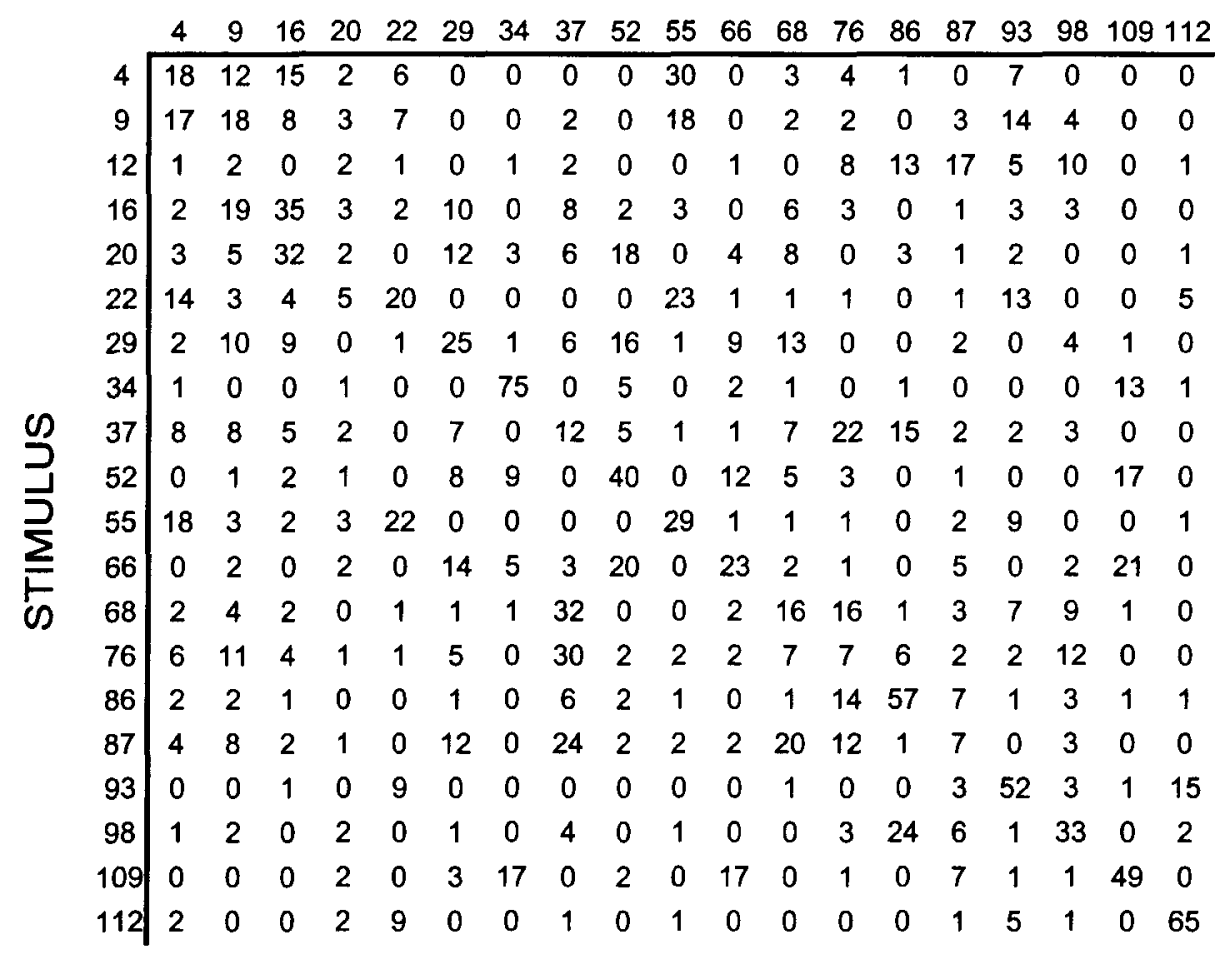

Note-Rows represent the texture shown, and columns the identification. Correct responses lie on the diagonal. The row and column labels are the Brodatz numbers of the textures. For Distance 2, boxes mark an example of mirrorposition cells, the sum totals of which are a measure of confusion of one texture for another (in this case, D37 sent/D68 received and D68 sent/D37 received).

(Manuscript received June 8, 1998;

revision accepted for publication May $28,1999$. 